\title{
Gradual opening of Smc arms in prokaryotic condensin
}

\section{Journal Article}

Author(s):

Vazquez Nunez, Roberto; Polyhach, Yevhen; Soh, Young-Min; Jeschke, Gunnar; Gruber, Stephan

Publication date:

2021-04-27

Permanent link:

https://doi.org/10.3929/ethz-b-000483991

Rights / license:

Creative Commons Attribution-NonCommercial-NoDerivatives 4.0 International

Originally published in:

Cell Reports 35(4), https://doi.org/10.1016/j.celrep.2021.109051 


\section{Cell Reports}

\section{Gradual opening of Smc arms in prokaryotic condensin}

\section{Graphical abstract}

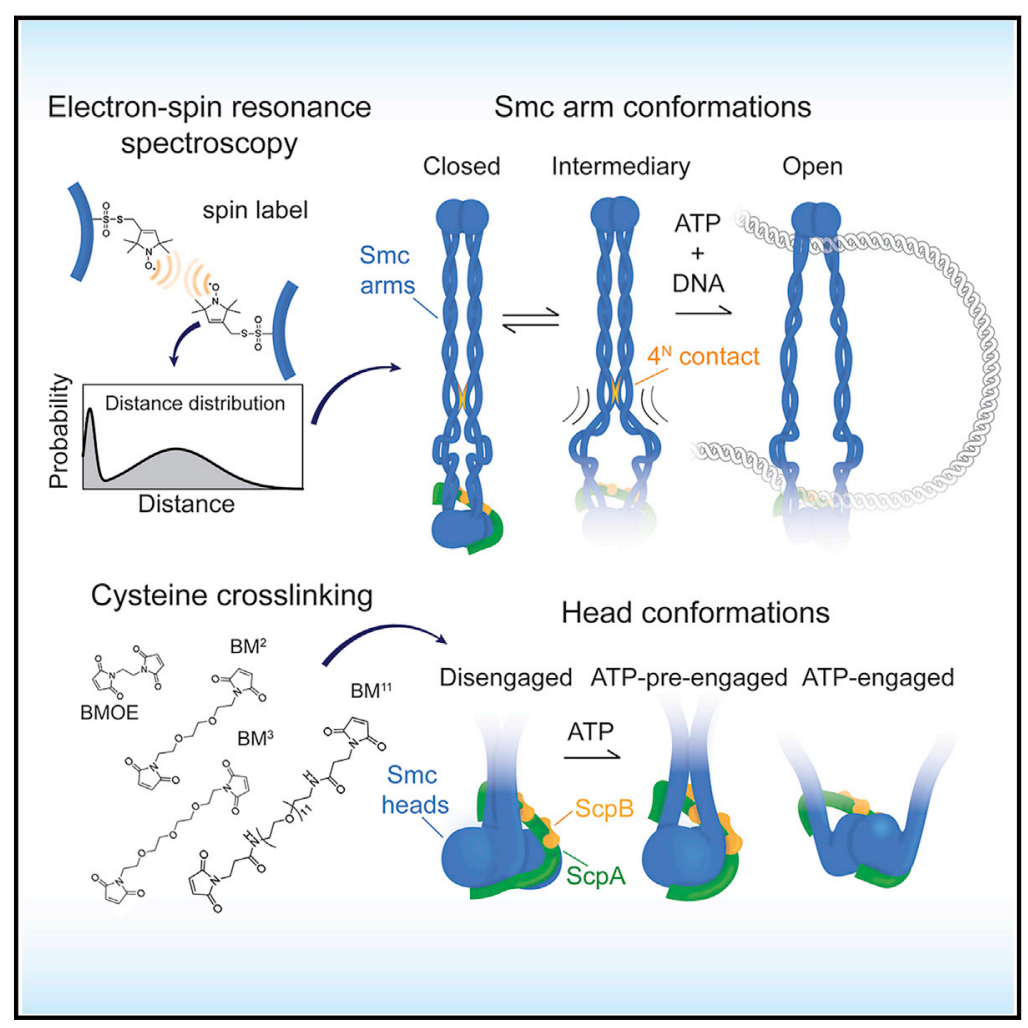

Highlights

- Smc arms partially open in absence of ATP and DNA

- ATP and DNA binding are essential for full opening of the arms

- Single-residue substitutions in the arm are lethal, likely by altering Smc dynamics

- Intermediary states may provide directionality to the Smc DNA translocation

\section{Authors}

Roberto Vazquez Nunez, Yevhen Polyhach, Young-Min Soh, Gunnar Jeschke, Stephan Gruber

Correspondence

stephan.gruber@unil.ch

\section{In brief}

Using electron-spin resonance spectroscopy and cysteine crosslinking, Vazquez Nunez et al. reveal intermediary conformations of the bacterial Smc complex. Smc arms partially open in the absence of ligands, and Smc heads adopt an ATP-pre-engaged state. Gradual opening of the Smc arms may direct DNA substrate binding. 


\title{
Article \\ Gradual opening of Smc arms in prokaryotic condensin
}

\author{
Roberto Vazquez Nunez, ${ }^{1,3}$ Yevhen Polyhach, ${ }^{2,3}$ Young-Min Soh, ${ }^{1}$ Gunnar Jeschke, ${ }^{2}$ and Stephan Gruber ${ }^{1,4, *}$ \\ ${ }^{1}$ Department of Fundamental Microbiology, University of Lausanne, 1015 Lausanne, Switzerland \\ 2Laboratory of Physical Chemistry, ETH Zurich, 8093 Zurich, Switzerland \\ ${ }^{3}$ These authors contributed equally \\ ${ }^{4}$ Lead contact \\ ${ }^{*}$ Correspondence: stephan.gruber@unil.ch \\ https://doi.org/10.1016/j.celrep.2021.109051
}

\section{SUMMARY}

Multi-subunit SMC ATPases control chromosome superstructure apparently by catalyzing a DNA-loopextrusion reaction. SMC proteins harbor an ABC-type ATPase "head" and a "hinge" dimerization domain connected by a coiled coil "arm." Two arms in a SMC dimer can co-align, thereby forming a rod-shaped particle. Upon ATP binding, SMC heads engage, and arms are thought to separate. Here, we study the shape of Bacillus subtilis Smc-ScpAB by electron-spin resonance spectroscopy. Arm separation is readily detected proximal to the heads in the absence of ligands, and separation near the hinge largely depends on ATP and DNA. Artificial blockage of arm opening eliminates DNA stimulation of ATP hydrolysis but does not prevent basal ATPase activity. We report an arm contact as being important for controlling the transformations. Point mutations at this arm interface eliminated Smc function. We propose that partially open, intermediary conformations provide directionality to SMC DNA translocation by (un)binding suitable DNA substrates.

\section{INTRODUCTION}

SMC protein complexes are ancient enzymes with a unique architecture that organize chromosomal DNA molecules, presumably by catalyzing DNA loop extrusion (Yatskevich et al., 2019). In eukaryotes, cohesin folds DNA into loop domains to regulate gene expression and to direct DNA recombination ( $\mathrm{Ba}$ et al., 2020; Merkenschlager and Nora, 2016). By a distinct mechanism, cohesin also holds sister chromatids together (Yatskevich et al., 2019). In mitosis, condensin folds DNA into a series of loops that are dynamically anchored along a chromatid axis, thus supporting chromosome condensation and sister chromatid resolution (Earnshaw and Laemmli, 1983; Gibcus et al., 2018; Marsden and Laemmli, 1979; Naumova et al., 2013). The essential functions of another relative, the Smc5/6 complex, are less well understood (Aragón, 2018).

In many bacteria, Smc-ScpAB complexes initiate a loopextrusion-type process at one or few selected starting points that are defined by 16-bp parS DNA sequences. parS sites are located in the replication origin region. They recruit the clamplike CTP-binding protein ParB (Jalal and Le, 2020; Osorio-Valeriano et al., 2019; Soh et al., 2019), which in turn promotes the loading of Smc-ScpAB complexes onto the chromosome (Gruber and Errington, 2009; Minnen et al., 2016; Sullivan et al., 2009; Wilhelm et al., 2015). Bidirectional translocation of Smc-ScpAB away from a parS site brings together the flanking DNA sequences, thus co-aligning the left and the right arm of the chromosome (Minnen et al., 2016; Tran et al., 2017; Wang et al., 2015, 2017). This translocation is thought to localize DNA entanglements on the replicating chromosome (i.e. knots and catenanes), facilitating chromosome individualization by DNA topoisomerase (Bürmann and Gruber, 2015; Gruber et al., 2014; Orlandini et al., 2019; Wang et al., 2014).

Smc-ScpAB complexes translocate rapidly ( $\sim 1 \mathrm{~kb} / \mathrm{sec})$ along chromosomal DNA in vivo. In vitro, they support only limited ATP hydrolysis activity $(<1 / \mathrm{sec})$, implying a large motor step size $(\sim 1$ kb or $600 \mathrm{~nm}$ ) (Hirano and Hirano, 2006; Vazquez Nunez et al., 2019; Wang et al., 2017, 2018). Several models for Smc translocation have been proposed (Diebold-Durand et al., 2017; Hassler et al., 2018; Marko et al., 2019; Terakawa et al., 2017). Smc proteins are comprised of an ABC-type ATP-binding head domain and a hinge domain connected at a distance by a long antiparallel coiled coil arm. Hinge domains form homotypic interactions in prokaryotic Smc complexes (Haering et al., 2002). Smc dimers furthermore associate with a kleisin subunit, in bacteria named ScpA. By its amino- and carboxy-terminal domains, ScpA bridges the head of one Smc protein with the head-proximal arm of the other (Bürmann et al., 2013). This generates tripartite SMC-kleisin rings that entrap chromosomal DNA double helices (Gligoris et al., 2014; Wilhelm et al., 2015). The kite subunit ScpB also forms dimers that associate with the central region of ScpA (Bürmann et al., 2013; Yatskevich et al., 2019).

Smc arms contact one another. They co-align lengthwise, thus collapsing the Smc-ScpAB complex into a rod-shaped particle (Diebold-Durand et al., 2017; Minnen et al., 2016; Soh et al., 2015). Eight distinct contacts are found between 
the two Smc arms, of which four involve amino-terminal sequences $\left(1^{\mathrm{N}}, 4^{\mathrm{N}}, 6^{\mathrm{N}}\right.$, and $8^{\mathrm{N}}$; see Figure $\left.2 \mathrm{~B}\right)$ and the other four carboxy-terminal sequences $\left(2^{\mathrm{C}}, 3^{\mathrm{C}}, 5^{\mathrm{C}}\right.$, and $7^{\mathrm{C}}$ ) (Diebold-Durand et al., 2017). In yeast condensin, corresponding sequences are also found in juxtaposition (Lee et al., 2020) (Figure S4D). In some SMC complexes (including MukBEF, cohesin, and condensin), the arms fold at an "elbow," thus bringing the hinge into proximity of the heads (Bürmann et al., 2019). Such folding has not yet been observed for bacterial Smc, and its role is unclear.

Smc heads engage with one another by sandwiching two ATP molecules using active site residues provided by both heads, thereby forming the catalytic center for ATP hydrolysis (Hirano et al., 2001; Hopfner, 2016; Lammens et al., 2004). ATP engagement of Smc heads is thought to be incompatible with full arm alignment (Diebold-Durand et al., 2017; Kamada et al., 2017; Lammens et al., 2004; Muir et al., 2020), thus delineating two mutually exclusive conformations, one with ATP-engaged heads (O-shaped "open" conformation) and one with completely aligned arms (I-shaped "closed" conformation). In the open conformation, ATP-engaged heads divide the lumen of the SMC-kleisin ring into the S compartment that is encircled by the long arms and the hinge and the $\mathrm{K}$ compartment that is enclosed by ScpAB. The open conformation of Bacillus subtilis (Bsu) Smc-ScpAB harbors two sites for DNA binding, namely, a hinge/DNA interface and a head/DNA interface (Vazquez Nunez et al., 2019). In the closed conformation, the Smc arms are aligned. The $S$ compartment is thus closed and separated from the $\mathrm{K}$ compartment by juxtaposed Smc heads. Principally consistent observations have recently been made by cryogenic electron microscopy for other SMC complexes, whereas atomic force microscopy (AFM) studies indicated much higher flexibility in the SMC arms (see Discussion). SMC arm dynamics appear crucial for DNA loop extrusion, but exactly how the different conformations bind to DNA and contribute to DNA translocation remains to be elucidated.

Here, we studied the shape of the Bsu Smc-ScpAB by electron paramagnetic resonance (EPR) spectroscopy. In addition to the open and the closed conformation, we observed an intermediary one with partially open S compartments. Separation of arms was detected near the heads even in the absence of ligands. Opening at the hinge, however, was found to largely depend on ATP and DNA binding. We showed that partial opening of the $S$ compartment is sufficient to support ATP hydrolysis, but intriguingly, DNA stimulation of the ATPase activity required complete opening. This implies that an open $\mathrm{S}$ compartment is an integral part of the normal ATP hydrolysis cycle. We identified one out of the eight arm contacts as being particularly important for controlling the dynamic Smc architecture. Mutating a single residue at this $4^{\mathrm{N}}$ contact interface eliminated Smc function, presumably by preventing arm closure. The mutations caused a defect in chromosome loading and DNA translocation. Altogether, our results suggest that the $\mathrm{S}$ compartment opens by a graded transition starting at the heads. Head-proximal arm contacts dissociate relatively easily, whereas hinge-proximal contacts are more stably engaged. The $4^{\mathrm{N}}$ contact appears critical for controlling the opening/closure reaction. It may provide directionality to the DNA translocation motor.

\section{RESULTS}

\section{Conformations of Smc-ScpAB detected by EPR}

Here, we aimed to characterize the conformational ensemble of Smc-ScpAB by measuring arm-to-arm distances. Based on published work, we expected the distances to be broadly distributed, covering a spectrum of closed and open conformations. Such dynamic structures are difficult to investigate by high-resolution techniques such as X-ray crystallography and electron microscopy. Therefore, we used EPR-double electron-electron resonance (DEER) that detects the coupling of electron spins over a larger distance range (from 1.5 to $10 \mathrm{~nm}$ ) and yields information on distance distributions for a population of particles (Jeschke, 2012; Polyhach et al., 2012; Reginsson and Schiemann, 2011). We labeled purified cysteine mutant Smc protein at one out of four selected positions (D193C, E217C, R643C, and R718C) with the methanethiosulfonate nitroxide spin label MTSL and reconstituted holo-complexes by mixing with unmodified ScpA and ScpB (Figure 1A). The EPR experiments were performed with the cysteine-lite $\mathrm{Smc}^{3 \mathrm{~S}}$ protein (C119S, C437S, and C826S) to minimize any off-target labeling. MTSL-labeling efficiencies were around $90 \%$, as calculated from continuouswave EPR spectra (Figure S1A). Of note, protein samples were prepared in a deuterated environment to reduce electron spin relaxation induced by proton nuclear spin diffusion (El Mkami and Norman, 2015). Deuterated buffers had only a minor impact on protein function, as judged by near-normal ATPase activity (Figure S1B). For most experiments, the Smc protein also included the Walker B E1118Q (EQ) mutation that hinders the ATP hydrolysis step. Protein preparations were pre-incubated with or without ATP and linear 40-bp double-stranded DNA, designated as dsDNA 40 , flash-frozen in liquid nitrogen, and measured at a temperature of $50 \mathrm{~K}$.

Most distances measured across the hinge domain dimer in $\mathrm{Smc}^{3 \mathrm{~S}}(\mathrm{R} 643 \mathrm{C}, \mathrm{E} 1118 \mathrm{Q})$ were narrowly distributed, with a peak at $\sim 1.5 \mathrm{~nm}$, which is at the lower edge of the sensitive range of high-power Q-band DEER and well-fitting with available crystal structures $(\mathrm{C} \alpha-\mathrm{C} \alpha \sim 1.1 \mathrm{~nm})$ (Figures $1 \mathrm{~A}$ and 1B; Griese and Hopfner, 2011; Haering et al., 2002; Kamada et al., 2017; Soh et al., 2015). A minor fraction with larger distances ( 4-6 nm) was potentially also noticeable (Figure 1B). Arm-to-arm distances near the heads showed a clear bimodal distribution in the absence of ligand (the apo state) (Figures 1B, S1C, S1E, and S1F). For Smc ${ }^{3 \mathrm{~S}}$ (D193C, $\mathrm{E} 1118 \mathrm{Q}$ ) (at contact $1^{\mathrm{N}}$ ), a population with narrowly distributed short distances, represented about $55 \%$ of all distances. These were centered at $\sim 1.7 \mathrm{~nm}$ and displayed a good fit with the Smc rod model built from crystal structures (Diebold-Durand et al., 2017). The long-distance population showed a much broader distribution spanning from about 3.0 to at least $6.0 \mathrm{~nm}$, indicating separated Smc arms near the heads in a substantial proportion of Smc complexes (Figure 1B). A similar pattern was observed with $\mathrm{Smc}^{3 \mathrm{~S}}$ (E217C, E1118Q) (at contact $2^{\mathrm{C}}$ ); a total of $\sim 69 \%$ of the dipolar couplings resided in a narrow short-distance population centered at $\sim 3.9 \mathrm{~nm}$, whereas other distances ranged from $\sim 6.0 \mathrm{~nm}$ to more than $8.5 \mathrm{~nm}$ (Figure 1B). Again, the short-distance population is in good agreement with the rod model (Diebold-Durand et al., 2017). For the hinge-proximal arm position $\mathrm{Smc}^{3 \mathrm{~S}}(\mathrm{R} 718 \mathrm{C}, \mathrm{E} 1118 \mathrm{Q})$ (at contact $\left.7^{\mathrm{C}}\right), 71 \%$ of the distances 
A

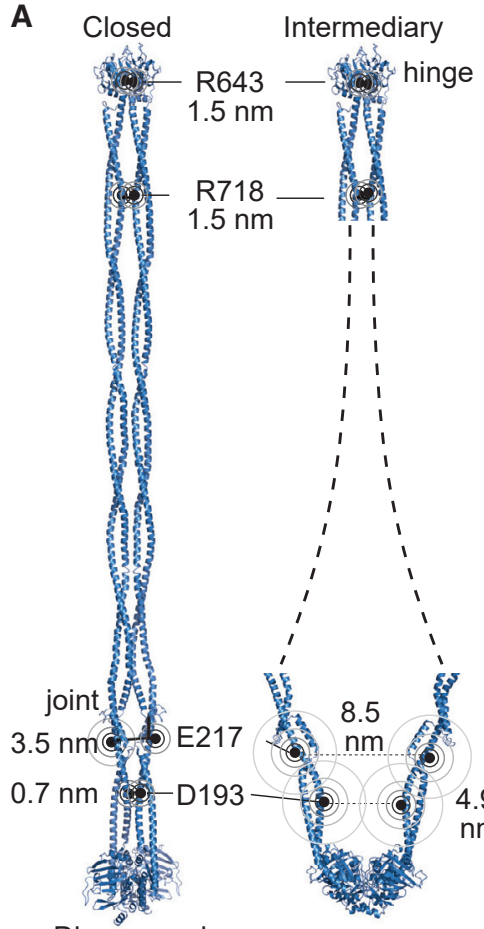

Disengaged heads

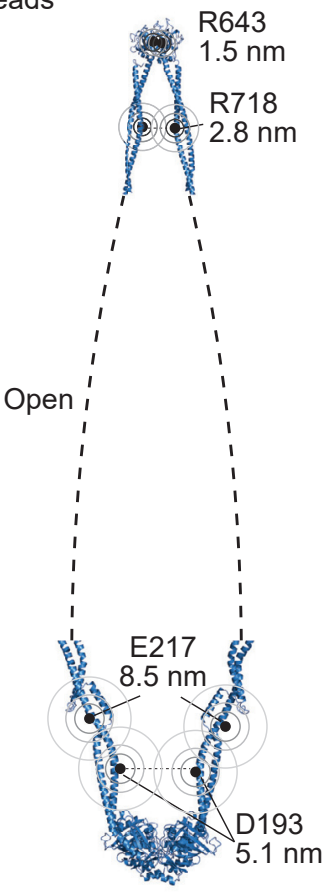

ATP-engaged heads
B

$\mathrm{Smc}^{3 \mathrm{~S}}(\mathrm{E} 1118 \mathrm{Q})-\mathrm{Scp} A \mathrm{~B}$, apo
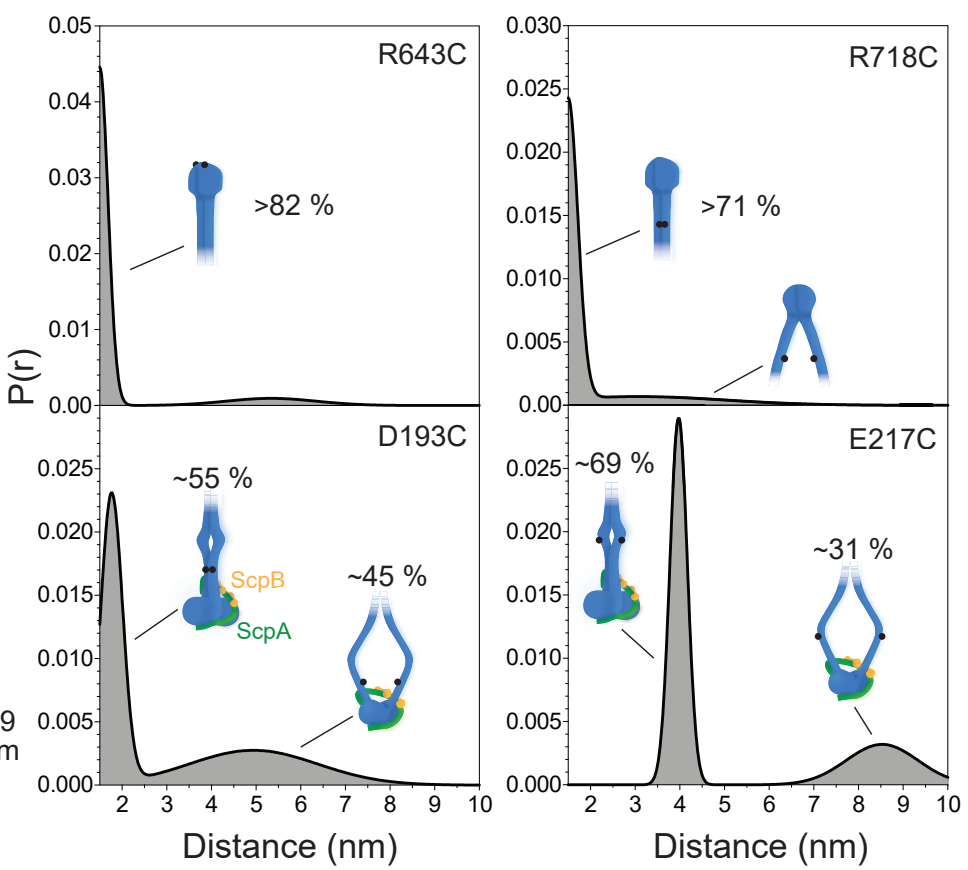

C

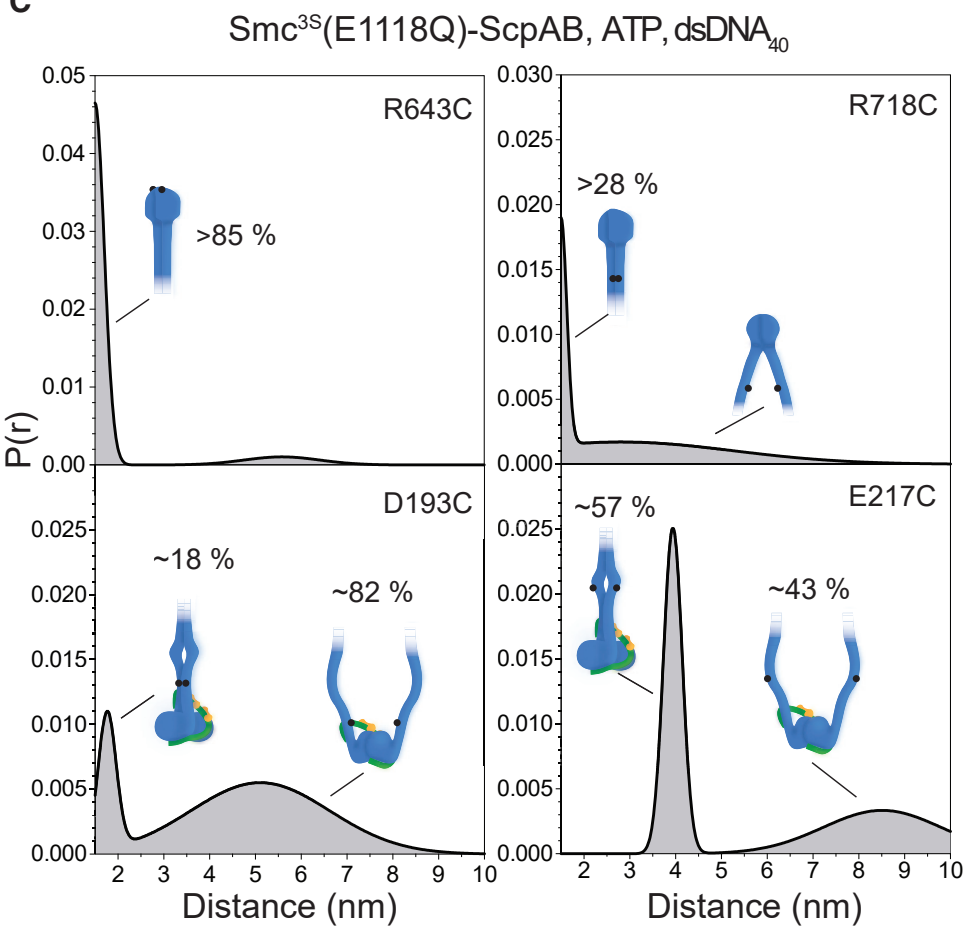

Figure 1. Distance distributions in Smc-ScpAB determined by electron spin resonance (EPR-DEER)

(A) Structural models of Smc dimers in the closed (top left panel), an intermediary (top right panel), and the open conformation (bottom panel). Models were built with the help of available crystal structures (Diebold-Durand et al., 2017; Soh et al., 2015). Dashed lines indicate putative bending of the Smc arms. Concentric circles indicate the positions of the cysteines used for MTSL labeling. Distances are maxima for sub-populations as observed by EPR.

(B) Probability distributions as measured by EPR-DEER with reconstituted Smc-ScpAB in the absence of ligands (apo). Schematics indicate the conformation for sub-populations with estimated relative abundance given.

(C) Probability distributions of Smc-ScpAB measured in the presence of $1 \mathrm{mM}$ ATP and $10 \mu \mathrm{M}$ 40-bp double-stranded DNA (dsDNA 40 ). Display as in (B). See also Figure S1 and Table S2. 
accumulated in a population with short arm-to-arm distances at $\sim 1.5 \mathrm{~nm}$. Similar to R643C, this number likely underestimates the size of the population due to partial suppression of the dipolar modulation at such short distances (Figure 1B). These measurements imply that in the absence of ligands one or more conformations exist in addition to the closed rod structure. Smc arms appeared separated near the heads (especially at contact $1^{N}$ ) in a larger fraction of complexes than near the hinge, implying the presence of partially open, intermediary conformations (Figure 1A). This is consistent with the pattern of arm cross-linking observed in vivo, where the levels of cysteine cross-linking were somewhat higher at contacts $8^{\mathrm{N}}$ and $6^{\mathrm{N}}$ than at contacts $3^{\mathrm{C}}, 2^{\mathrm{N}}$, and $1^{\mathrm{C}}$ (Diebold-Durand et al., 2017).

The addition of ATP to Smc(E1118Q)-ScpAB did not change the distribution between the short- and long-distance population for S217C and R643C (Figure S1D). For D193C, the fraction of long distances became slightly larger, indicating a trend toward arm opening upon ATP binding. For S217C, the long distances became somewhat shorter. The distance distribution at the hinge (R643C) remained virtually unchanged also in the presence of ATP and DNA (Figure 1D). Arm-to-arm distances, however, showed a pronounced shift from the short-distance to the now predominant long-distance population upon pre-incubation with ATP and DNA. For example, close to the hinge (R718C) (at contact $7^{\mathrm{C}}$ ), a broad long-distance population ranging from about 2.0 to $7.0 \mathrm{~nm}$ represented about $70 \%$ of all measurable distances in the presence of ATP and DNA (Figure 1C). The extent of shift to larger distances varied slightly between the cysteine positions, possibly resulting from uncertainty in quantifying short-distance $(<0.5 \mathrm{~nm})$ or longdistance $(>10 \mathrm{~nm})$ populations or indicating that the cysteine residues or their chemical labeling mildly affected the stability of the conformations. Regardless, these results provided strong support for the notion that Smc arms separate from one another upon ATP and DNA addition. The Smc arms are presumably fully detached in a significant fraction of complexes when bound to ATP and DNA. From the distance distribution at R718C, we estimated that the arms are connected to the hinge at narrow angles ( 0 to $\sim 45^{\circ}$ ). More open angles-as seen in some crystal structures of Smc hinge fragments $\left(\sim 180^{\circ}\right)$-were however not observed. The open conformation thus represents elongated, oval-shaped particles. Of note, we obtained similar trends in the absence of ScpAB and when using wild-type (WT) Smc instead of Smc(E1118Q) proteins (Figures S1E and S1F), although arm dissociation (at D193C) with ATP and DNA was less pronounced with WT Smc than with Smc(E1118Q).

Altogether, we conclude from the EPR measurements that apo Smc-ScpAB exists mainly in the closed conformation and in smaller sub-populations of the open and very likely also of an intermediary conformation. Upon ligand binding, the distributions shift toward larger arm-to-arm distances, generating a sizeable fraction of open conformations. The broad range of measured distances indicates that the arms are somewhat flexible, at least when arm alignment is lost.

Partial arm opening is sufficient for ATP hydrolysis, but DNA stimulation requires full opening

We next wondered whether intermediary conformations-as observed by EPR-are able to support ATP hydrolysis or whether a fully open conformation is a prerequisite for ATP hydrolysis. To test this, we blocked the $S$ compartment opening by engineering a covalent arm-to-arm junction at selected arm positions, namely, at contacts $1^{\mathrm{N}}, 3^{\mathrm{C}}, 4^{\mathrm{N}}$, and $7^{\mathrm{C}}$ (Figure 2B). Stiff arms would be expected to prevent ATP hydrolysis when conjoined, whereas flexible arms would support normal ATP hydrolysis even when conjoined. A reduction in the ATPase rate varying with the position of the engineered arm-to-arm junction would imply limited flexibility.

We again made use of site-directed chemical modification. We cross-linked cysteine residues in purified preparations of Smc with the thiol-reactive compound 1,3-propanediyl-bismethanethiosulfonate (M3M) to then reconstituted arm-linked holo-complexes and measured their ATPase activity (Figure 2A). M3M cross-linking is efficient as well as reversible, thus allowing the engineered arm-to-arm connection to break to recover lost activity. These experiments were performed with the cys-free $\mathrm{Smc}^{3 \mathrm{SV}}$ (C119S, C437S, C826S, and C1114V) protein (Figure 2C). However, comparable results were obtained with the $\mathrm{Smc}^{3 \mathrm{~S}}$ variant (Figure S2A). Incubation of $\mathrm{Smc}^{3 S \mathrm{~V}}$ (D193C), $\mathrm{Smc}^{3 \mathrm{SV}}$ (Q320C), $\mathrm{Smc}^{3 \mathrm{SV}}(\mathrm{A} 715 \mathrm{C})$, or Smc ${ }^{3 \mathrm{SV}}$ (Y944C) with M3M produced at least $70 \%$ to $85 \%$ of chemically cross-linked dimers, as judged by non-reducing SDS-PAGE analysis. An addition of up to $10 \mathrm{mM} \mathrm{DTT}$ was required to reverse the cross-linking reaction (Figure $2 \mathrm{C}$ ).

All cross-linked preparations yielded significant ATPase activity regardless of the position of the engineered arm-to-arm junction. Although a Cys-free control sample showed no discernible effect of M3M treatment on ATP hydrolysis (Figure 2C), a substantial loss of ATPase activity was observed with head-proximal cysteine residues (D193C at contact $1^{\mathrm{N}}$ and $\mathrm{Y} 944 \mathrm{C}$ at contact $3^{\mathrm{C}}$ ). No such effect was seen for the more distant positions (Q320C and A715C at contacts $4^{\mathrm{N}}$ and $7^{\mathrm{C}}$, respectively). In all cases, normal ATPase activity was restored by pre-incubation with a reducing agent. These results strongly suggest that complete separation of Smc arms is not required for productive head engagement and for ATP hydrolysis. Intermediary conformations may support ATP hydrolysis. Cross-linking of Q320C (at contact $4^{\mathrm{N}}$ ) even increased the basal ATPase rate (see below).

We found that the stimulation of the ATPase activity by addition of dsDNA 40 was eliminated by M3M treatment regardless of the position of the cysteine residue on the arm and regardless of any dampening or stimulating effect of the arm-to-arm junction on ATP hydrolysis (Figure 2B). DNA stimulation was however restored upon pre-incubation with a reducing agent. The stimulation of the Smc ATPase by dsDNA 40 thus depends on the separation of residues on opposite arms, which is consistent with the idea that complete arm opening is required for DNA stimulation. DNA binding at the hinge/DNA interface may boost ATP hydrolysis by modifying the organization of the arms, as previously proposed (Soh et al., 2015). While strongly inhibiting DNA-stimulated ATP hydrolysis activity (Figure 2D), the arm-toarm junction at $\mathrm{Y} 944 \mathrm{C}$ did not substantially hinder DNA binding by Smc-ScpAB (Figure S2B), suggesting that ATP hydrolysis is uncoupled from DNA binding in the cross-linked material or that DNA binding predominantly occurs at a site this is uncoupled from the ATPase also in unmodified Smc-ScpAB. 
A
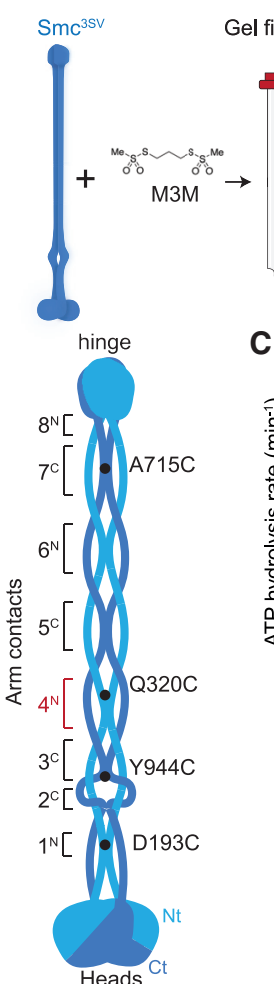

C
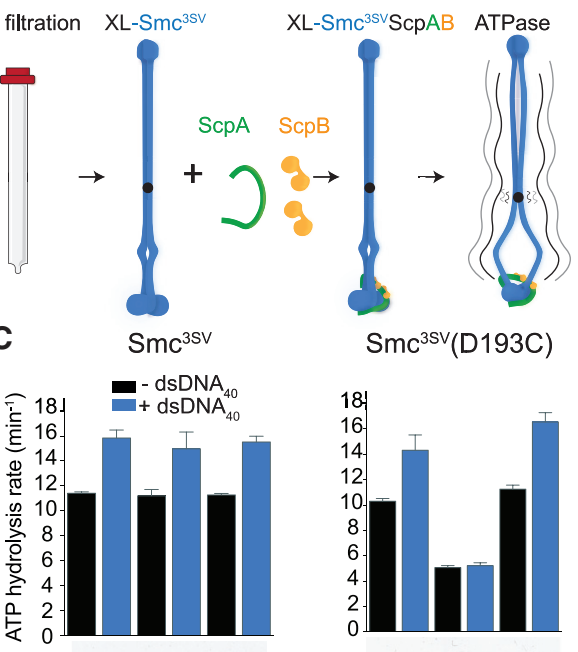

$\mathrm{Smc}^{3 \mathrm{~Sv}}(\mathrm{D} 193 \mathrm{C})$
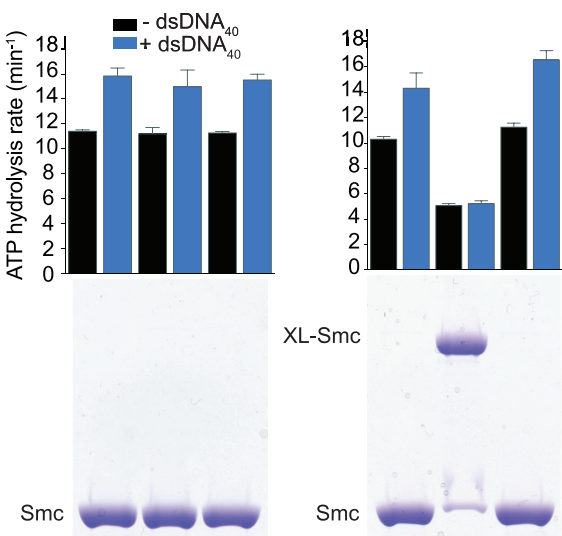

$\mathrm{XL}-\mathrm{Smc}$ DMSO M3M M3M M3M
DTT $\mathrm{Smc}^{3 \mathrm{SV}}(\mathrm{Q} 320 \mathrm{C})$ $\mathrm{Smc}^{3 \mathrm{SV}}(\mathrm{Y} 944 \mathrm{C})$
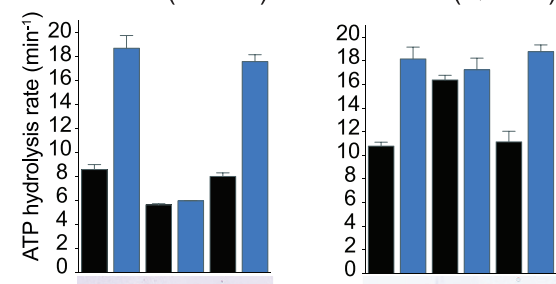

$\mathrm{XL}-\mathrm{Smc}$

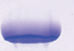

$\mathrm{XL}-\mathrm{Smc}$
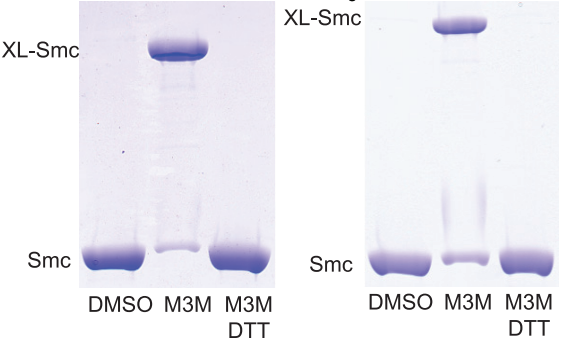
$\mathrm{Smc}^{3 \mathrm{SV}}(\mathrm{D} 193 \mathrm{C})$
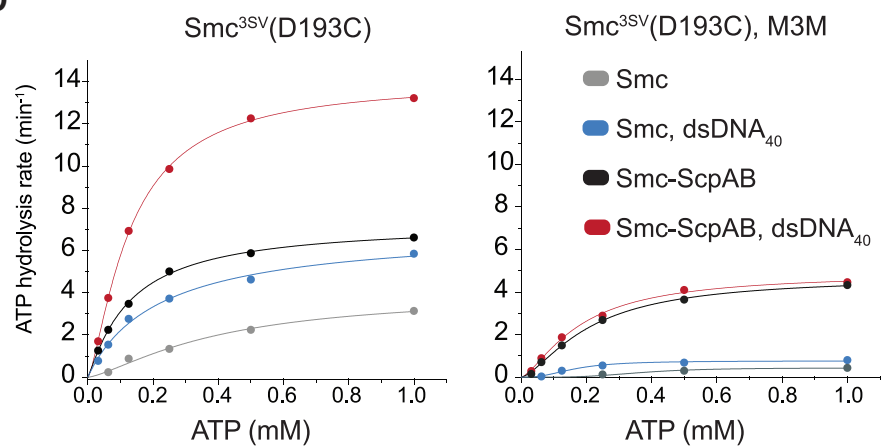

Figure 2. ATP hydrolysis by Smc-ScpAB with cross-linked arms

(A) Schematic for the preparation of cross-linked protein for ATPase measurements. Single cysteine $\mathrm{Smc}^{3 \mathrm{SV}}$ proteins were purified and incubated with M3M cross-linker. Excess of M3M and any protein aggregates were removed by gel filtration. Eluate fractions were mixed with stoichiometric amounts of purified ScpA and $\mathrm{ScpB}$ to reconstitute holo-complexes. ATPase activity was determined.

(B) Positions of arm contacts and engineered cysteine residues in the Bacillus subtilis (Bsu) Smc dimer in the closed conformation (DieboldDurand et al., 2017). Amino-terminal sequences (Nt) are shown in lightblue colors and carboxy-terminal sequences $(\mathrm{Ct})$ in dark-blue colors. Inter-subunit arm contacts are numbered 1 to 8 from the heads toward the hinge. Superscripts ( $\mathrm{N}$ or $\mathrm{C}$ ) indicate the involvement of amino- or carboxy-terminal sequences at the contact, respectively. Black circles denote the positions of cysteine residues engineered for site-specific crosslinking.

(C) ATP hydrolysis rates for preparations of Smc-ScpAB with crosslinked arms. Top panels: bar graphs of ATP hydrolysis rates per Smc protein measured with $1 \mathrm{mM}$ ATP; $0.15 \mu \mathrm{M}$ Smc-ScpAB complex in the absence (black bars) and presence (blue bars) of $3 \mu \mathrm{M}$ dsDNA 40 . Error bars correspond to the standard deviation calculated from three technical replicates. Bottom panels: Coomassie-stained SDS-PAGE gel of cross-linked Smc protein. DMSO, solvent only, M3M, crosslinker only; DTT, cross-linker with subsequent treatment with reducing agent.

(D) ScpAB dependence of the ATP hydrolysis rates of cross-linked $\mathrm{Smc}^{3 \mathrm{SV}}$ (D193C). Hydrolysis rates were measured at $0.15 \mu \mathrm{M}$ protein concentration in the presence and absence of stoichiometric amounts of ScpAB and $3 \mu \mathrm{M}$ dsDNA 40 . Left panel: non-crosslinked control; right panel: M3M cross-linked proteins. Lines represent non-linear regression fits to the Hill model.

See also Figure S2 and Tables S3 and S4. 


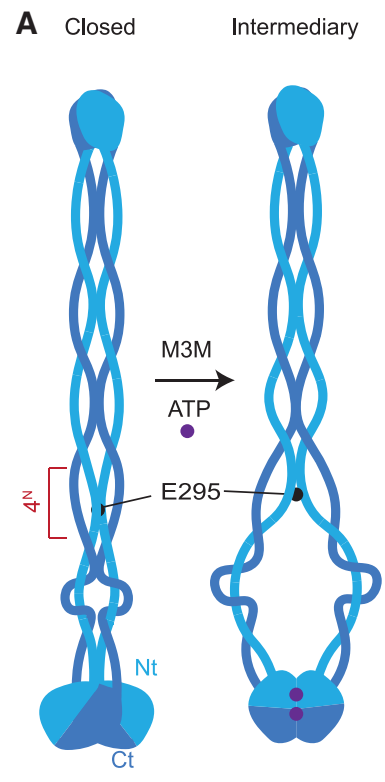

B PDB: 5NNV

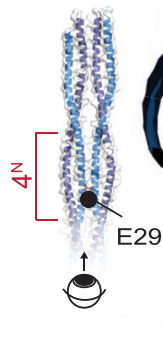

C

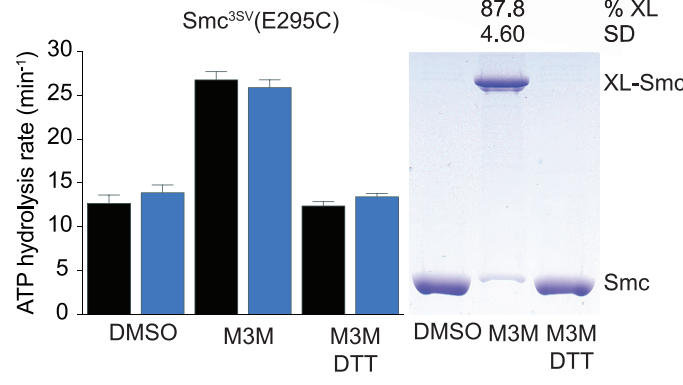

Figure 3. Cross-linking of E295C at the $4^{\mathrm{N}}$ arm contact enhances ATP hydrolysis

(A) Schematic of arm contacts in the Smc dimer in closed and intermediary conformations. The position of E295 is indicated as a black dot, and the $4^{\mathrm{N}}$ arm contact is denoted by a red bracket. ATP molecules are represented as purple dots.

(B) Structural organization of the $4^{\mathrm{N}}$ arm contact in Bsu Smc (PDB: 5NNV) in cartoon representation. Hydrogen bonds are indicated as dotted lines in yellow colors.

(C) ATP hydrolysis rates and SDS-PAGE of crosslinked Smc(E295C). Display as in Figure 2C.

See also Figure S3 and Table S4.

Cross-linking of E295C may further disrupt Smc rod alignment. Intriguingly, we previously isolated point mutations at the $4^{\mathrm{N}}$ arm contact (i.e. D280G, Q320R, or E323K) that suppressed the lethal phenotype of an arm-length-modified Smc protein (Bürmann et al., 2017), supporting the notion that the $4^{\mathrm{N}}$ arm contact

Altogether, these findings imply that the Smc ATPase can operate in the following two modes: as a basal ATPase in a partially open conformation and as a DNA-stimulated ATPase, presumably with an open conformation. The separation of head-proximal arms promotes ATP hydrolysis, whereas hinge-proximal arms may remain juxtaposed during basal ATP hydrolysis cycles. Interestingly, ScpAB becomes essential for ATP hydrolysis when arms are conjoined at contact $1^{\mathrm{N}}$ (D193C) (Figure 2D), suggesting that it helps to overcome constraints imposed by the engineered junction on the ATPase heads. Of note, the fact that DNA is unable to stimulate ATP hydrolysis in arm-locked complexes is consistent with the prior observation that the hinge/DNA interface but not the head/DNA interface is important for DNA stimulation of ATP hydrolysis (Vazquez Nunez et al., 2019).

\section{Stimulation of ATP hydrolysis by chemical modification} of Smc arms

During the above experiments, we noticed that another cysteine variant, $\mathrm{Smc}^{3 \mathrm{SV}}(\mathrm{E} 295 \mathrm{C})$, showed aberrant enzyme kinetics. The protein supported robust cysteine cross-linking ( $87.8 \% \pm 4.6 \%)$, with the cross-linked protein preparation displaying a $\sim 2.5$-fold higher ATPase activity (Figures 3C, S3A, and S3B). This increase was reversed to normal levels by pre-incubation with a reducing agent. The E295C mutation largely eliminated DNA stimulation of the ATPase even without cross-linking. This finding confirmed that robust ATP hydrolysis can be achieved when arms are covalently conjoined (at contact $4^{\mathrm{N}}$ ) and suggests that Smc arms can have a positive effect on ATP hydrolysis even when they are artificially linked together.

In the crystal structure (PDB: 5NNV), E295 residues form inter-subunit hydrogen bonds with S294 residues (Figure 3B), thus likely stabilizing the $4^{\mathrm{N}}$ arm contact (Diebold-Durand et al., 2017). The lack of these hydrogen bonds in the E295C mutant presumably interferes with normal arm alignment. is particularly important for controlling conformational transitions during the ATP hydrolysis cycle.

A critical contact for arm alignment

To discern the physiological relevance of the $4^{\mathrm{N}}$ arm contact, we next looked at the conservation of Smc arm sequences at this interface. Residue G302 and neighboring residues are well conserved in firmicute Smc proteins, whereas arm sequences are otherwise relatively poorly conserved (Figure 4A). The glycine residue is located directly at the interface (Figure 4B), separated by two $\alpha$-helical turns from E295. To test whether G302 is important for Smc function, we generated 10 substitutions by allelic replacement at the endogenous smc locus in Bsu (Figure 4C). Remarkably, substitution to glutamate (GE), tryptophan (GW), or phenylalanine (GF) prevented growth of Bsu on nutrient-rich medium-similar to smc deletion mutants-despite the GE and GW proteins being expressed at normal levels (Figure 4D). Three other mutants (substitution to methionine [GM], glutamine [GQ], and lysine [GK]) were unable to support growth of a $\triangle$ parB mutant on this growth medium, whereas the arginine mutant (GR) only poorly supported growth of the double mutant (Figure S4A). Substitutions for residues with smaller side chains (alanine, valine, and serine) had no discernible effects on growth. Thus, residues with negatively charged or bulky sidechains at position 302 hinder protein function, presumably by destabilizing the $4^{\mathrm{N}}$ arm contact. To test this, we combined the G302 mutations with a sensor cysteine for arm alignment (A715C, at contact $7^{\mathrm{C}}$ ) and one for head juxtaposition (S152C). In these experiments, we focused on two G302 mutants with a severe growth defect and sidechains with distinct physicochemical properties (GE and GW). The two mutations indeed reduced cysteine cross-linking at both sites in vivo, with GW having rather mild and GE quite dramatic effects (Figure 4E). The GW mutation also strongly reduced the abundance of the closed conformation (i.e., the short distance population), as measured by EPR with 
A

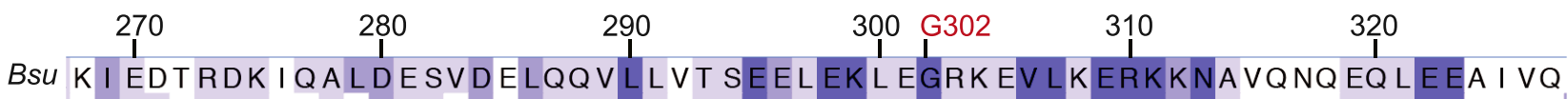
Gst H I EQ LRDQMAA I DES I DGLQQVLLLA SEELEKLEGRKEV LKERKKNAEKRKEQLEETAAA Spn YLQKQKTQRQKLERDTDHTN I DL I T T T EEYEKF I GK LNVLEERKRNQSET NARFEEELDN Sau QI QQYKGKRHQLDNDVESLNYQLVKATEA FEKYT GQ LNVLEERKKNQSET NARYEEEQEN

B

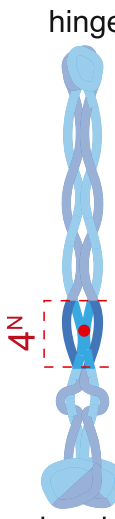

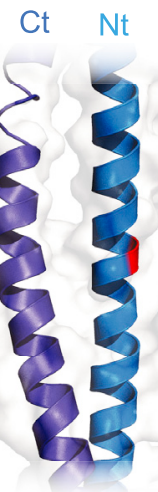

$\mathrm{Nt} \quad \mathrm{Ct}$

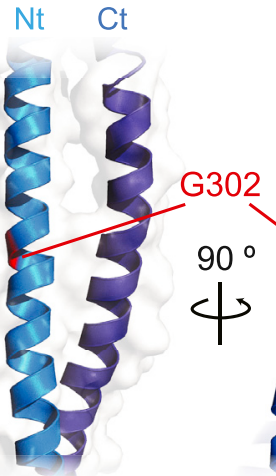

$\mathrm{Nt} \quad \mathrm{Nt}$

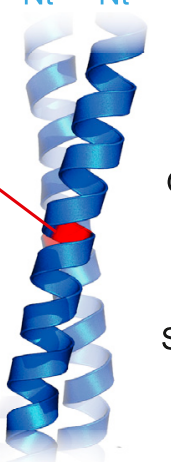

C

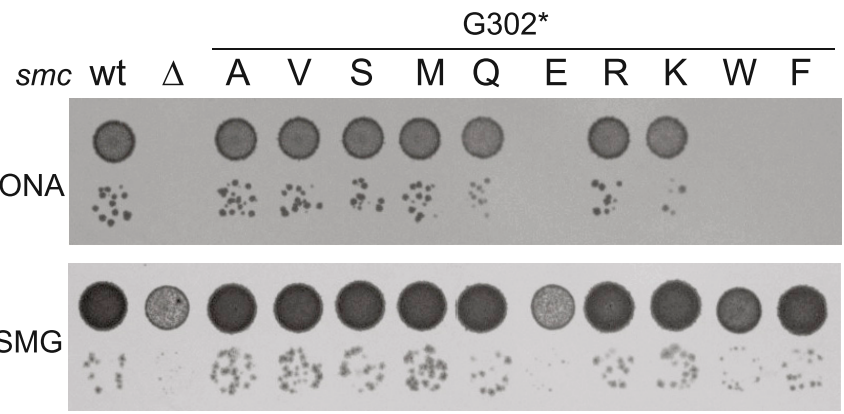

E

D

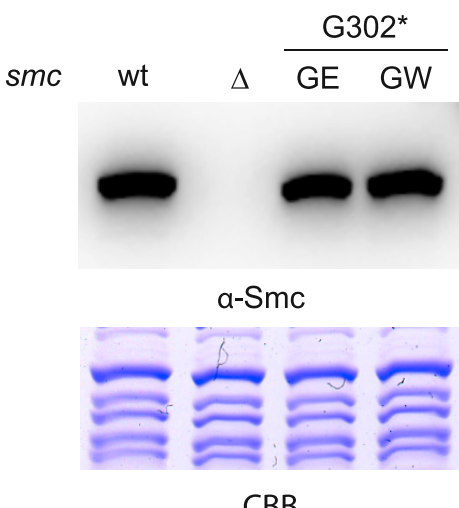

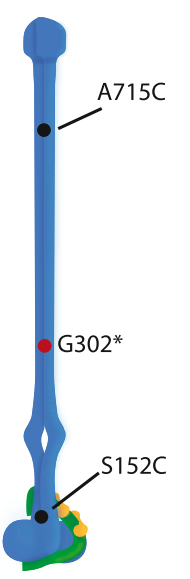
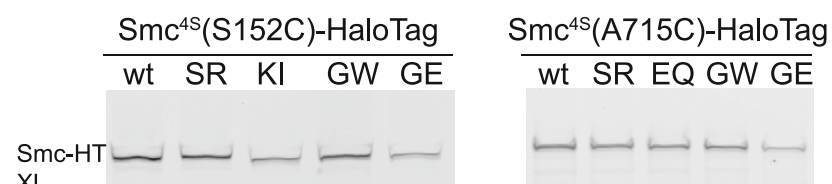

$\mathrm{XL}$

$\mathrm{Smc-HT} \square \square$

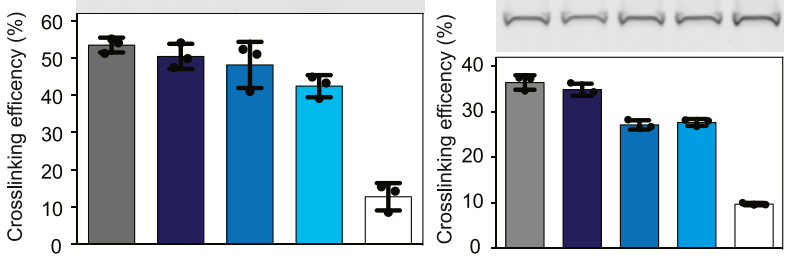

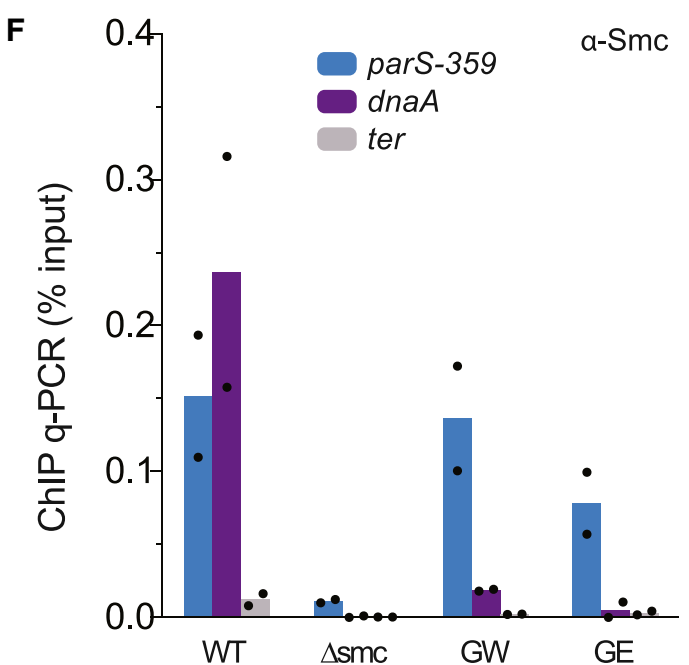

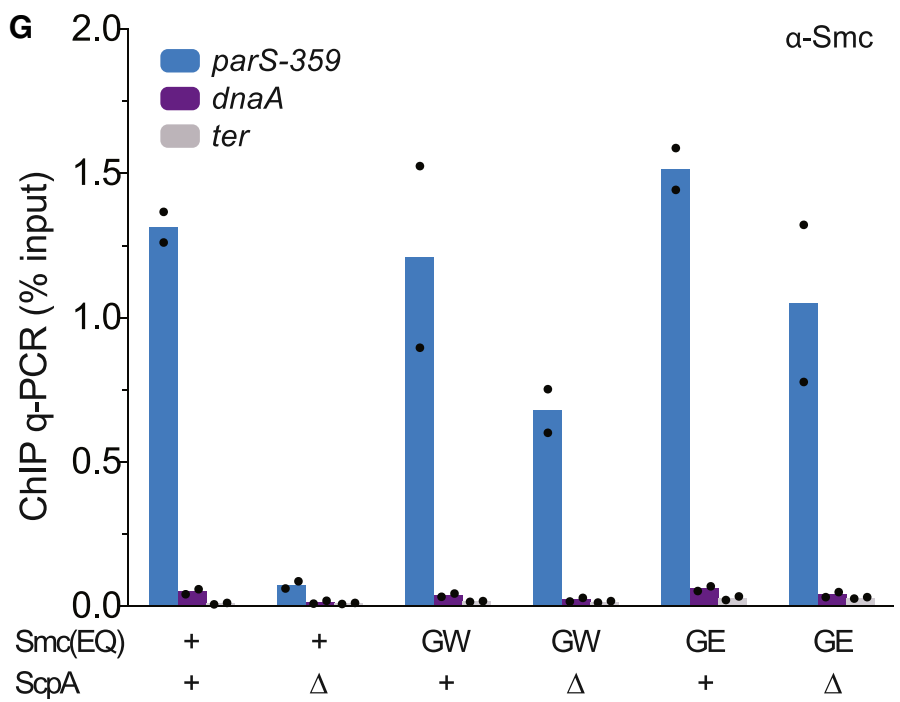


D193C in the absence and presence of ATP and DNA (Figure S4B; Table S2). We conclude that residue G302 and the $4^{\mathrm{N}}$ arm contact are important for co-aligning arms in the closed conformation.

\section{Defective chromosome loading of $4^{\mathrm{N}}$ arm contact mutants}

We next determined the chromosomal distribution of Smc(GE) and $\mathrm{Smc}(\mathrm{GW})$ proteins by chromatin immunoprecipitation (ChIP) by using $\alpha$-ScpB and $\alpha$-Smc antisera. Both mutants had close to normal enrichment at the parS-359 site but very low enrichment at the dnaA locus, as determined by ChIP-qPCR (Figure 4F). These mutants thus appear to target normally to parS but then fail to load onto DNA or to translocate along DNA (Minnen et al., 2016). Consistent with this notion, we found that their E1118Q variants showed elevated levels of enrichment at parS, which were similar or even higher than the otherwise WT $\mathrm{Smc}(\mathrm{E} 1118 \mathrm{Q})$ protein. Formation of the $4^{\mathrm{N}}$ arm contact may thus be required for the loading reaction or for the release of Smc protein from ParB/parS loading sites. Notably, a putative ParB/Smc interface has been mapped onto the Smc arm in the vicinity of the $3^{\mathrm{C}}$ and $4^{\mathrm{N}}$ arm contacts (Minnen et al., 2016).

We found that the GW and the GE mutations alleviated the requirement for $\mathrm{ScpA}$ in targeting of the $\mathrm{Smc}(\mathrm{E} 1118 \mathrm{Q})$ protein to a parS site (Figure 4G). ScpA may thus contribute to overcoming constraints imposed by the aligned Smc arms to facilitate the transition to the open conformation for parS targeting (Minnen et al., 2016). The G302 mutations likely alleviate these constraints and eliminate the requirement for ScpAB. The integrity of the $4^{\mathrm{N}}$ arm contact is subsequently required for the closure of the $S$ compartment and the clearance of Smc-ScpAB from ParB/parS. Purified preparations of $\mathrm{Smc}(\mathrm{G} 302 \mathrm{~W})$ and to a weaker extent also Smc(G302E) displayed increased ATP hydrolysis activity, which was curiously hindered rather than enhanced by the addition of ScpAB and DNA (Figure S4C). These point mutations in the Smc arms thus lead to mis-regulation of the ATPase by DNA.

\section{Intermediary conformations in vivo}

We next focused on the organization of Smc heads in vivo by inferring their conformations from patterns of cross-linking ob- tained with $\mathrm{Smc}(\mathrm{K} 1151 \mathrm{C})$. The $\mathrm{K} 1151 \mathrm{C}$ residue was previously engineered to detect the ATP-engaged state $(\mathrm{C} \alpha-\mathrm{C} \alpha$ distance: $11.6 \AA$ ) by bismaleimidoethane (BMOE) cross-linking (Minnen et al., 2016). Here, we made use of bis-maleimide cross-linkers with longer spacers - which supported robust cross-linking in intact Bsu cells - to detect alternative conformations (Figure 5A). We observed that cross-linking of Smc(K1151C)-HaloTag was much more efficient with 1,11-bismaleimido-triethyleneglycol $\left(\mathrm{BM}^{3} ; 17.8 \AA\right)$ and bis-MAL-dPEG11 $\left(\mathrm{BM}^{11} ; 62.3 \AA\right)$ than with BMOE (8 $\AA$ ) or 1,8-bismaleimido-diethyleneglycol $\left(\mathrm{BM}^{2} ; 14.7 \AA\right)$ (Figures 5A and S5A) (Minnen et al., 2016). The Walker A ATPbinding mutant $\mathrm{Smc}(\mathrm{K} 37 \mathrm{I})$ also showed an upward trend in cross-linking with extending spacer length, but the cross-linking efficiencies did not reach levels comparable to those of WT Smc (Figure $5 \mathrm{~A})$. The $\mathrm{Smc}(\mathrm{E} 1118 \mathrm{Q})$ protein displayed roughly equivalent cross-linking efficiencies regardless of spacer length (Figures 5A and S5A). Finally, a clear downward trend in crosslinking with increasing spacer length was observed when the E1118Q mutation was combined with three different Smc arm alterations (Figure 5B). For example, the $\mathrm{Smc}(204 \Omega / 996 \Omega$, E1118Q) protein-harboring a 13-amino acid peptide insertion in each arm-showed most efficient cross-linking with BMOE and decreasing efficiencies with increasing spacer length (Diebold-Durand et al., 2017). A similar trend was observed with an artificially shortened "mini"'Smc(E1118Q) protein (Bürmann et al., 2017) and with Smc(GE, E1118Q) (Figure 5B). This downward trend in cross-linking was expected for the ATP-engaged state because the $\mathrm{K} 1151 \mathrm{C}$ residues are closely juxtaposed and thus ideally positioned for cross-linking by the smallest compound BMOE (Minnen et al., 2016).

We conclude that in addition to two previously known states (disengaged and ATP-engaged) (Figure 1A), Smc heads also occur in a third, hitherto uncharacterized state. We envision that in this "ATP-pre-engaged" state, active site residues from both heads are aligned (thus supporting efficient K1151C cross-linking by $\mathrm{BM}^{3}$ and $\mathrm{BM}^{11}$ ), but the closure of the catalytic pocket remains incomplete due to a persisting gap between the signature motif residues from one head and the ribonucleotide bound to the opposing head (thus reducing K1151C cross-linking by BMOE). Taking all in vivo cross-linking data into account (Figures 5A and 5B), we estimated the occupancy of the different

Figure 4. Residue G302 at the $4^{\mathrm{N}}$ arm contact is essential for Smc function

(A) Alignment of $4^{\mathrm{N}}$ arm contact sequences from selected firmicute species (Gst, Geobacillus stearothermophilus; Spn, Streptococcus pneumoniae; Sau, Staphylococcus aureus). Residues G302 is indicated.

(B) The position of residue G302 at the $4^{\mathrm{N}}$ arm contact (PDB: $5 \mathrm{NNV}$ ). Left panel: schematic of arm contact zones as in Figure $2 \mathrm{~B}$; right panel: structural view of the $4^{\mathrm{N}}$ arm contact from Bsu Smc in cartoon representation in front and side views. Colors as in Figure 3B. G302 is shown in red colors.

(C) Colony formation by Bsu strains with single-amino acid substitution in G302. $9^{2}$-fold and $9^{5}$-fold dilutions of stationary-phase cultures were spotted on nutrient-rich medium (ONA) and nutrient-poor minimal glucose-glutamate medium (SMG) and grown at $37^{\circ} \mathrm{C}$ for 16 and $24 \mathrm{~h}$, respectively.

(D) Smc protein levels in mutants G302E (GE) and G302W (GW) grown in SMG medium determined by immunoblotting with polyclonal antibodies raised against Bsu Smc protein. Coomassie staining (CBB) of cell extracts on separate gels is shown as control for uniform protein extraction.

(E) Quantification of cross-linked species of Smc ${ }^{4 S}$-HaloTag (C119S, C437S, C826S, and C1114S) harboring G302 mutations (labeled with TMR-HaloTag ligand). Cysteine pairs were introduced at reporter positions S152 (left panels) and A715 (right panels). Cross-linking efficiencies were calculated by in-gel fluorescence quantification of band intensities of non-crosslinked (Smc-HT) and crosslinked (Smc-HT XL) species. Efficiencies were compared between wild-type (WT), the ABC signature motif mutant S1090R (SR), the ATP-binding mutant K37I (KI), and G302W (GW) and G302E (GE) mutants. Dots represent data points from three biological replicates. Mean and standard deviations are indicated as boxes and bars, respectively.

(F) Chromatin immunoprecipitation coupled to quantitative PCR (ChIP-qPCR) in Bsu cells by using $\alpha$-Smc antiserum. Selected loci near the replication origin and at the terminus regions were analyzed. Dots represent data points from one out of two biological replicates. Mean values are indicated as bars.

(G) ChIP-qPCR by using $\alpha$-Smc antiserum. As in (F) for strains carrying the E1118Q (EQ) mutation and lacking or having scpA.

See also Figure S4. 
A

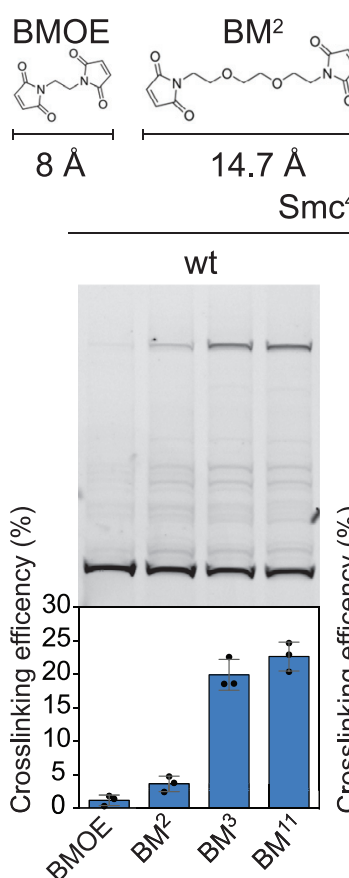

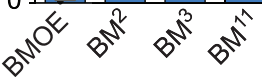

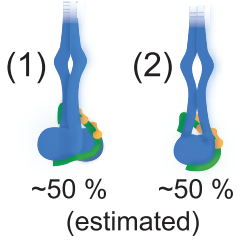

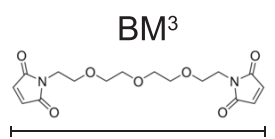
$17.8 \AA$
(K1151C)-HaloTag
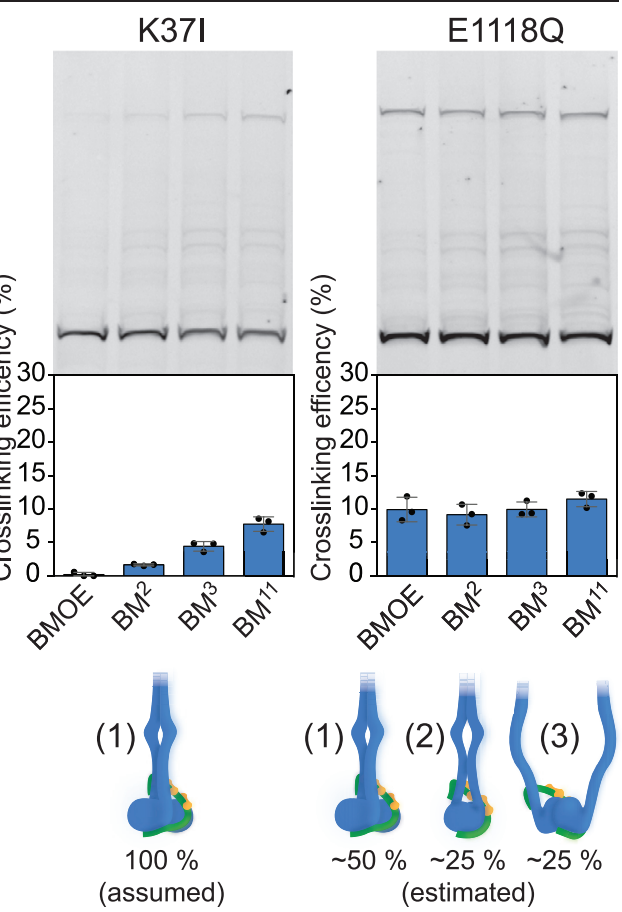

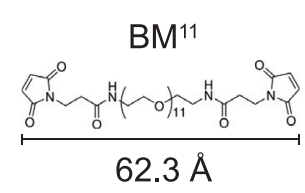

$62.3 \AA$

$1118 Q$

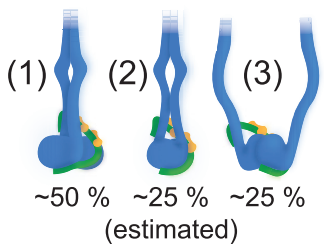

C

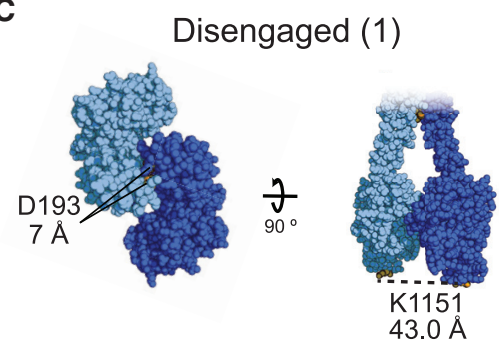

ATP-pre-engaged (2)

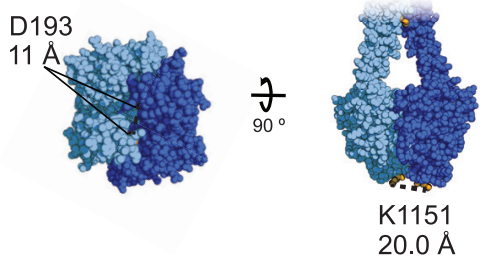

ATP-engaged (3)

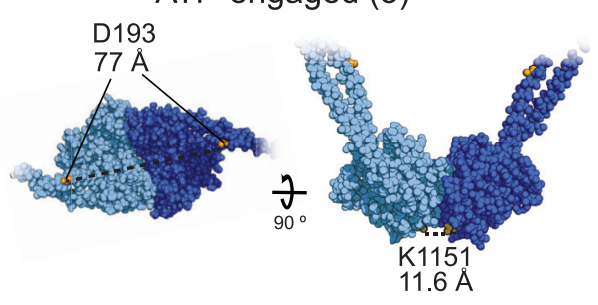

B

$\mathrm{Smc}^{4 \mathrm{~S}}(\mathrm{~K} 1151 \mathrm{C})$-HaloTag
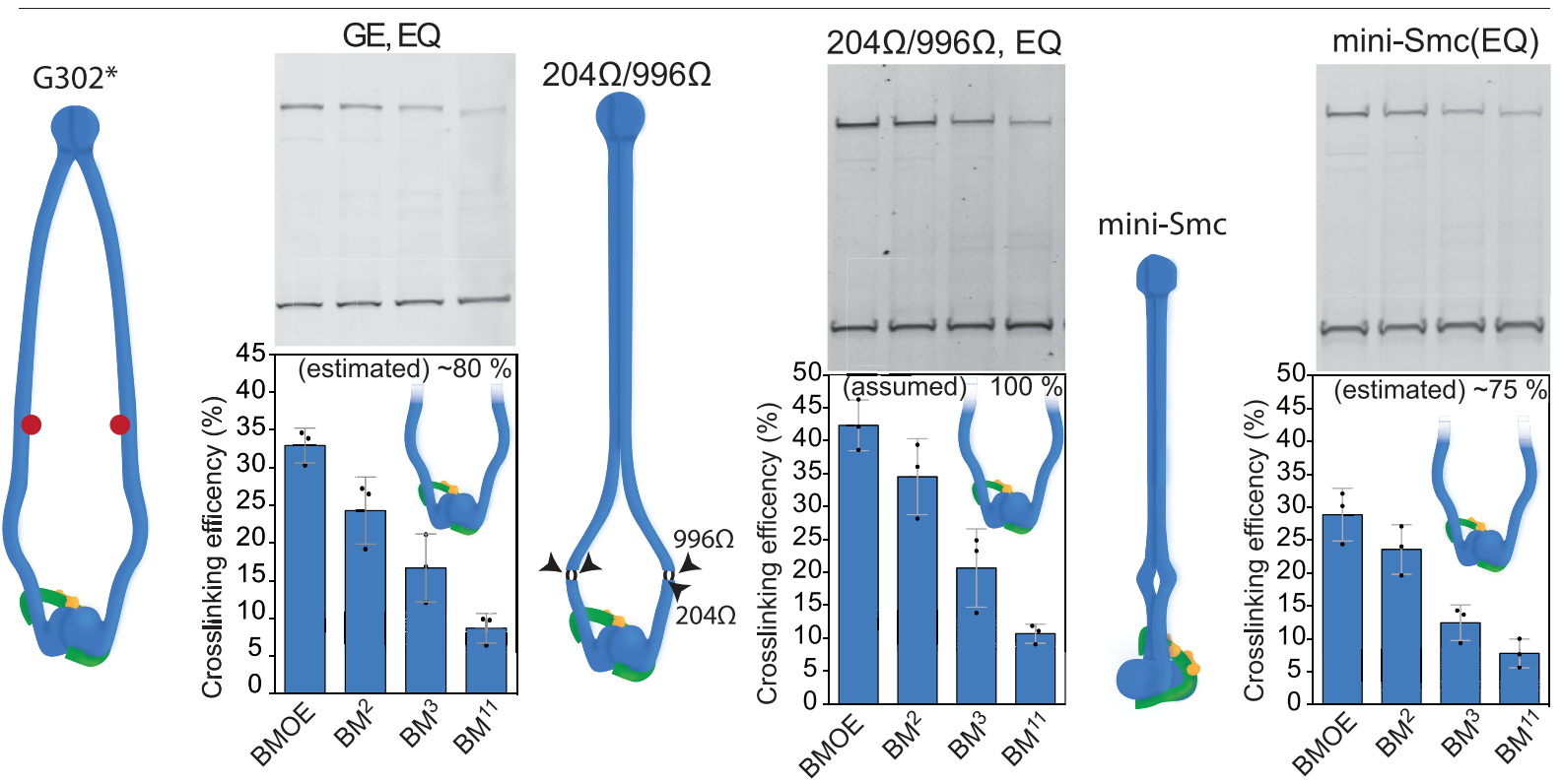
states in WT Smc. Assuming a 100\% occupancy of the ATP-en-

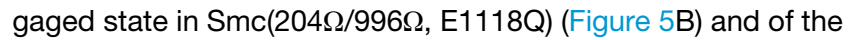
disengaged state in the ATP-binding mutant Smc(K37I) (Figure $5 A$ ), we suggest that WT Smc-ScpAB complexes rarely populated the ATP-engaged state and distributed roughly equally between the other states (Figure $5 A$ ). In Smc(E1118Q), about a one-fourth of complexes appear to be ATP-engaged, suggesting that head engagement is an inefficient and stepwise process in vivo, unless arm integrity is affected.

Of note, although ScpA is crucial for forming the ATP-engaged state in Smc(E1118Q), the requirement for ScpA was (partially) alleviated in the GW and GE variants (Figure S5E) (Minnen et al., 2016). As mentioned above, ScpA was dispensable for the targeting of the GE and GW Smc(E1118Q) proteins to parS sites (Figure 4G), which is consistent with the notion that ScpAB helps to disrupt arm co-alignment to facilitate head engagement and parS targeting. This is in excellent agreement with the efficient and ScpA-independent parS targeting of a hinge dimerization mutant of Smc(EQ), which also displays mis-aligned Smc arms and increased levels of head engagement (Minnen et al., 2016).

Finally, we wanted to establish whether the ATP-preengaged state-detected by $\mathrm{BM}^{3}$ and $\mathrm{BM}^{11}$ cross-linking of Smc(K1151C)-corresponded to the closed, the open, or the intermediary conformation. Using a combination of cysteine residues, we found that arm cross-linking with Q320C (at contact $4^{\mathrm{N}}$ ) appeared to occur largely independently of K1151C cross-linking, implying that the $4^{\mathrm{N}}$ arm contact is mostly engaged when heads are cross-linked at K1151C (Figure 6A). The efficient $\mathrm{BM}^{3}$ cross-linking of ATP-pre-engaged heads (in otherwise WT Smc) thus must have occurred on a (largely) closed conformation, implying that the closed conformation exists in a disengaged and an ATP-pre-engaged state (Figure 6B).

\section{DISCUSSION}

Elucidating the mechanism underlying DNA loop extrusion requires an understanding of the conformational transitions in SMC complexes during the ATP hydrolysis cycle, as well as the concomitant DNA binding and unbinding events. Here, we provide structural insights into three separate conformations and the corresponding state of the ATPase. The conformational dynamics appears largely governed by a limited rigidity of the head-proximal Smc arms and by a finite stability of arm contacts. Although the architecture appears dynamic, principles of organization can be deduced.

The conformational landscape of SMC complexes Cryoelectron microscopy (cryo-EM) recently helped to reveal the molecular architecture of SMC-kleisin complexes (in apo and ATP-engaged states) from eukaryotes (Collier et al., 2020; Higashi et al., 2020; Lee et al., 2020; Shi et al., 2020). EPR is ideally suited to close remaining gaps by providing information on the distributions of flexible and dynamic conformations (Jeschke, 2018; Kazmier et al., 2014; Vercellino et al., 2017).

\section{The closed conformation}

Independent lines of evidence point to the prevalence and conservation of a closed conformation in SMC complexes. It is predominant in reconstituted Smc-ScpAB, as judged by EPR (Figure 1), and in living Bsu cells according to site-specific cross-linking experiments (Diebold-Durand et al., 2017). Its main characteristic features are disengaged heads and completely aligned arms. Point mutations that hinder arm alignment eliminate Smc function in Bsu, implying that the closed conformation is critically important (Figure 4). Yeast condensin was found by cryo-EM as an elongated particle with "nonengaged" heads and completely aligned arms (Lee et al., 2020), presumably corresponding to the Smc-ScpAB rod, albeit displaying folded Smc2 and Smc4 arms (see below). Intriguingly, the Smc2 and Smc4 arms in apo condensin are most closely juxtaposed at the region that corresponds to the $4^{\mathrm{N}}$ arm contact in Smc-ScpAB, which is consistent with the idea that this contact has retained its special function in yeast condensin, and possibly other SMC complexes too (Figure S4D).

The condensin arms bend $\sim 180^{\circ}$ at an elbow, allowing the hinge to fold back and contact the middle of the Smc2 arm (Lee et al., 2020). This feature has not been observed for Smc-ScpAB and may have evolved in eukaryotic SMC proteins (and independently also in MukBEF) (Bürmann et al., 2019). The function of arm folding and of the hinge/arm contact is not well understood. It is conceivable that the folding may be relevant to the process of stepwise opening and closing of the $\mathrm{S}$ compartment, possibly stabilizing half-open states and thus providing additional levels of control. Of note, the closed conformation is rare in AFM images of yeast condensin and Bsu Smc-ScpAB, unless the material is cross-linked prior to

Figure 5. Smc head cross-linking in vivo

(A) Top panel: chemical structures of bis-maleimido crosslinkers with variable spacer arms (BMOE, bismaleimidoethane; BM ${ }^{2}, 1,8$-bismaleimido-diethyleneglycol; $\mathrm{BM}^{3}, 1,11$-bismaleimido-triethyleneglycol; $\mathrm{BM}^{11}$, bis-MAL-dPEG11). Bottom panels: quantification of cross-linked species of Smc ${ }^{4 S}$ harboring the K1151C cysteine pair reporter by using different crosslinkers. Crosslinking efficiencies were calculated as described for Figure 4E and compared between WT (left panel), K37I (middle panel), and EQ (right panel) variants. Error bars were calculated as standard deviation from three biological replicates. The values and schematics shown below the graphs indicate the approximately estimated relative abundance of different conformations, based on assumed abundances for the

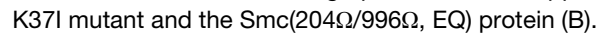

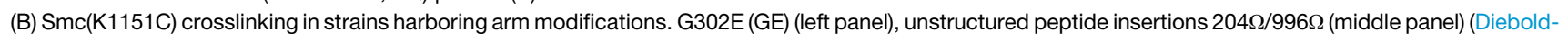
Durand et al., 2017), and mini-Smc CC298 (Bürmann et al., 2017) (right panel). Representation and quantification as in (A).

(C) Structural models of different ATPase states. Model for the disengaged state (1) as described in Diebold-Durand et al. (2017). The ATP-engaged state (3) is based on a crystal structure (PDB: 5XG3). The ATP-pre-engaged state (2) is manually built to aligning ABC motifs without opening the arms. Individual subunits are colored in light or dark blue colors, respectively. D193 and K1151 inter-subunit $\mathrm{C} \alpha-\mathrm{C} \alpha$ distances are shown as a reference for the different conformations.

See also Figure S5. 
A

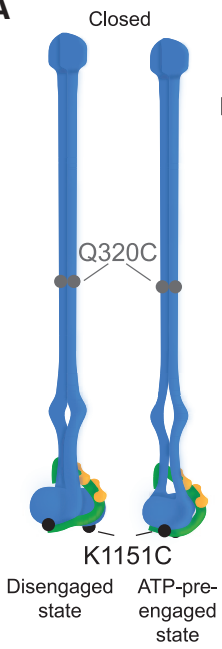

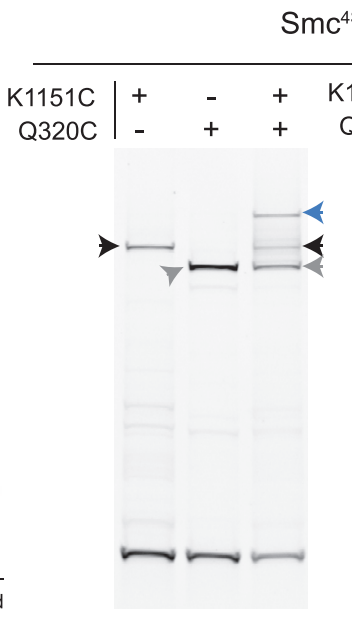

$\mathrm{Smc}^{4 \mathrm{~S}}$-HaloTag, $\mathrm{BM}^{3}$

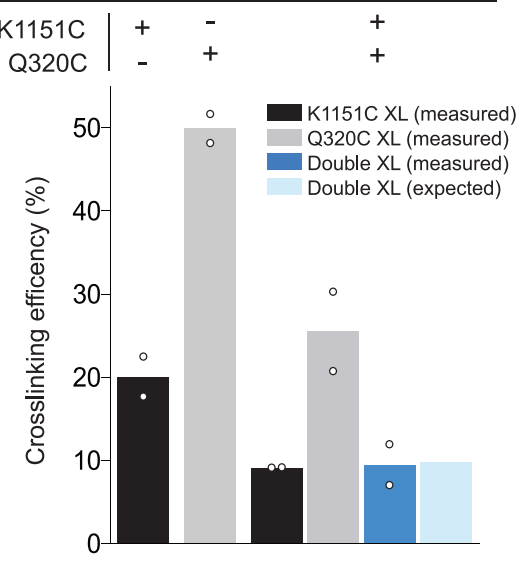

B

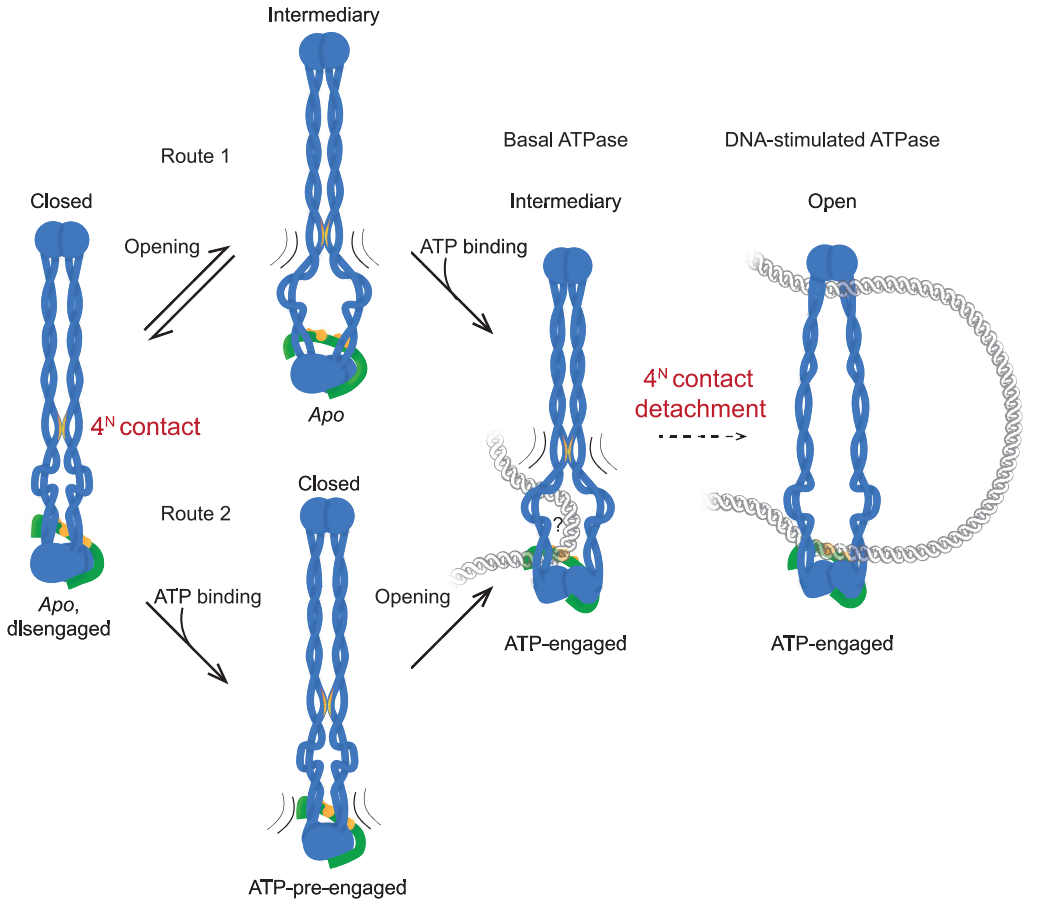

Figure 6. Conformational states and transitions in Smc-ScpAB

(A) Simultaneous cross-linking of Bsu Smc heads and arms in vivo. Left panel: schematics of Smc-ScpAB in closed conformations with disengaged or ATP-preengaged heads. Cysteine reporters Q320C and $\mathrm{K} 1151 \mathrm{C}$ are indicated as dots in black and gray colors, respectively. Right panel: quantification of single and double cross-linking efficiencies in $\mathrm{Smc}^{4}$. HaloTag with Q320C and/or K1151C. The gray arrowhead denotes the species cross-linked only at Q320C; black denotes species only cross-linked at $\mathrm{K} 1151 \mathrm{C}$, and blue denotes species cross-linked at Q320C and K1151C. Individual data points are shown as white circles from two biological replicates. Average values are shown as bars (same color coding as for arrowheads). Data are also shown in Figure S6. (B) Model of the conformational landscapes and multistep opening of Smc-ScpAB. Two alternative pathways are present in the basal ATPase. Route 1: spontaneous separation of the juxtaposed heads leads to an intermediary conformation with constraint arms, which is then stabilized by ATP binding and head engagement. Route 2: ATP binding in the closed conformation generates ATP-pre-engaged heads, which are constrained by the arms. Partial arm opening and head engagement result in the intermediary conformation. Optional: full opening of the arms produces the DNA-stimulated ATPase.

See also Figure S6.

integral part of the DNA-stimulated ATP hydrolysis cycle. Smc dimers that are unable to completely open the $S$ compartment retained the ability to hydrolyze ATP but lost the stimulation upon DNA addition. Hinge/ DNA binding may open the arms at the hinge as previously proposed (Soh et al., 2015), thus facilitating the transition to ATPengaged heads and supporting efficient ATP hydrolysis.

\section{Intermediary conformations}

Here, we have expanded our knowledge on the conformational landscape of Smc-ScpAB by detecting and characterizing intermediary conformations using three complementary

sample deposition (Fuentes-Perez et al., 2012; Ryu et al., 2019; Yoshimura et al., 2002). We suspect that the nonspecific adsorption of samples onto the AFM mica may affect SMC rod folding dynamics.

\section{The open conformation}

The open conformation of Smc-ScpAB has previously been inferred indirectly from crystal structures of isolated ATP-engaged heads and supported by low-resolution images obtained by rotary shadowing electron microscopy and by loss of cysteine cross-linking (Anderson et al., 2002; Diebold-Durand et al., 2017; Kamada et al., 2017; Lammens et al., 2004). In this study, we provide direct evidence for the existence of the open conformation. We characterize its shape and show that it represents an approaches (EPR, ATPase measurements, and cysteine crosslinking) (Figures 1, 2, and 5). Altogether, our data suggest that Smc heads and the head-proximal arms undergo dynamic structural changes.

Patterns of cross-linking demonstrate that the Smc heads can adopt at least three different states in vivo, here denoted as disengaged, ATP-pre-engaged, and ATP-engaged state (Figure $6 \mathrm{~B})$. The ATP-engaged state occurs in the open and the intermediary conformation. We propose that in the ATP-pre-engaged state, the active site residues from both heads are aligned by ATP but the arms remain (largely or completely) aligned. The heads may transition gradually from the disengaged, by the ATP-pre-engaged to the ATP-engaged state. Both steps might be impeded by arm alignment. 
Artificially engineered arm junctions reduce-but do not block-ATP hydrolysis (Figures 2 and 3). The ATP-engaged state-presumably a prerequisite for ATP hydrolysis - is thus compatible with partial opening of arms. Whether such basal ATPase cycles are futile or contributing to DNA translocation remains to be established. The fact that the essential head/ DNA interfaces is exposed in the intermediary conformation (while the non-essential hinge/DNA interface is likely inaccessible) is consistent with the notion that this state is functionally relevant (Figure 6B; Vazquez Nunez et al., 2019).

Cryo-EM structures of DNA-bound cohesin in the ATPengaged state show a partially open conformation with separated head-proximal arms (Collier et al., 2020; Higashi et al., 2020; Shi et al., 2020). Most of the more distal arm sequences are less well resolved-probably due to structural flexibilitybut appear to remain aligned (and elbow folded) (Collier et al., 2020; Higashi et al., 2020). Whether conformations with completely dissociated arms are present in cohesin (and condensin) is not fully established, but rotary-shadowing electron micrographs would be consistent with this notion (Anderson et al., 2002). Upon arm dissociation, the arms either remain folded at the elbow, thus producing B-shaped structures as inferred from AFM images of condensin (Eeftens et al., 2016), or they unfold at the elbow to generate O-shaped complexes (as seen by EM and similar to the open conformation of SmcScpAB).

The intermediary conformations of Smc-ScpAB are structurally not well defined, probably due to significant flexibility in the head-proximal arms. In condensin, however, a well-defined partially open state was detected by cryo-EM. This apo bridged conformation of yeast condensin showed partial separation of arms and heads (Lee et al., 2020). The separated heads are however bridged-and likely stabilized-by one of two hawk subunits, which are specific to condensin and cohesin. Our observations, together with the recent cryo-EM structures, indicate that intermediary conformations (with partially opened arms) are widely conserved in SMC complexes.

The DNA translocation cycle

It is tempting to speculate that the intermediary conformations control the process of compartment opening and closure and direct the binding and unbinding of suitable DNA substrates, thus driving directional DNA translocation. Here, we propose that one of the eight arm contacts acts as a molecular switch for arm opening in response to initial DNA contact (Figure 6B). Chromosomal DNA is entrapped in the $\mathrm{K}$ compartment of the closed conformation, as determined by site-specific crosslinking in Bsu Smc-ScpAB (Vazquez Nunez et al., 2019), and in yeast cohesin (Chapard et al., 2019). The open conformation holds two DNA binding interfaces in the $S$ compartment, of which the head/DNA interface-but not the hinge/DNA interface-is essential for Smc function and critical for Smc DNA translocation (Hirano and Hirano, 2006; Vazquez Nunez et al., 2019). These observations suggest that DNA is transiently occupying the $S$ compartment. How DNA enters and exits the $S$ compartment is a crucial open question.

The loop-capture model proposes that DNA is inserted as a loop on top of the heads and upon ATP hydrolysis exits the $S$ compartment by transfer to the $\mathrm{K}$ compartment between the heads (Diebold-Durand et al., 2017; Marko et al., 2019). Alternatively, the DNA enters the S compartment by transfer between the heads, as recently proposed for yeast cohesin (Collier et al., 2020). How the latter may lead to DNA translocation and DNA loop extrusion is however unclear. Unilateral and gradual opening of the arms-as described in this study-is likely directly relevant to this key substrate selection process. Future experiments will have to establish how the conformations (open, closed, and intermediary) of SMC complexes associate with chromosomal DNA double helices to promote DNA loop extrusion.

\section{STAR $\star$ METHODS}

Detailed methods are provided in the online version of this paper and include the following:

- KEY RESOURCES TABLE

- RESOURCE AVAILABILITY

O Lead contact

- Materials availability

O Data and code availability

- EXPERIMENTAL MODEL AND SUBJECT DETAILS

$\bigcirc$ Bacillus subtilis strains and growth

Protein purification

- METHOD DETAILS

○ ATPase assay

- M3M crosslinking-ATPase assay

Fluorescence anisotropy measurements

Double electron-electron resonance (EPR-DEER)

In vivo cross-linking

Chromatin immunoprecipitation

- QUANTIFICATION AND STATISTICAL ANALYSIS

Analysis of cross-linking efficiencies

O Steady-state kinetics

O DEER traces analysis

O Analysis of qPCR Data

\section{SUPPLEMENTAL INFORMATION}

Supplemental information can be found online at https://doi.org/10.1016/j. celrep.2021.109051.

\section{ACKNOWLEDGMENTS}

We thank Frank Bürmann for help in setting up initial EPR-DEER experiments and for helpful comments on the manuscript and Daniel Klose for collecting EPR-DEER data. We are grateful to all members of the Gruber laboratory for continuous support and stimulating discussions. This work was supported by the European Research Council (Horizon 2020 ERC CoG 724482).

\section{AUTHOR CONTRIBUTIONS}

Conceptualization, R.V.N., Y.P., and S.G.; investigation, R.V.N., Y.P., and Y.-M.S.; data curation, methodology, validation, and formal analysis, R.V.N.; writing and visualization, R.V.N., Y.P., G.J., and S.G.; supervision and project administration, S.G.; and funding acquisition, S.G. and G.J. 


\section{DECLARATION OF INTERESTS}

The authors declare no competing interests.

Received: October 13, 2020

Revised: January 20, 2021

Accepted: April 7, 2021

Published: April 27, 2021

\section{REFERENCES}

Anderson, D.E., Losada, A., Erickson, H.P., and Hirano, T. (2002). Condensin and cohesin display different arm conformations with characteristic hinge angles. J. Cell Biol. 156, 419-424.

Aragón, L. (2018). The Smc5/6 Complex: New and Old Functions of the Enigmatic Long-Distance Relative. Annu. Rev. Genet. 52, 89-107.

Ba, Z., Lou, J., Ye, A.Y., Dai, H.Q., Dring, E.W., Lin, S.G., Jain, S., Kyritsis, N., Kieffer-Kwon, K.R., Casellas, R., et al. (2020). CTCF orchestrates long-range cohesin-driven V(D)J recombinational scanning. Nature 586, 305-310.

Bürmann, F., and Gruber, S. (2015). SMC condensin: promoting cohesion of replicon arms. Nat. Struct. Mol. Biol. 22, 653-655.

Bürmann, F., Shin, H.C., Basquin, J., Soh, Y.M., Giménez-Oya, V., Kim, Y.G., Oh, B.H., and Gruber, S. (2013). An asymmetric SMC-kleisin bridge in prokaryotic condensin. Nat. Struct. Mol. Biol. 20, 371-379.

Bürmann, F., Basfeld, A., Vazquez Nunez, R., Diebold-Durand, M.L., Wilhelm, L., and Gruber, S. (2017). Tuned SMC Arms Drive Chromosomal Loading of Prokaryotic Condensin. Mol. Cell 65, 861-872.e9.

Bürmann, F., Lee, B.G., Than, T., Sinn, L., O’Reilly, F.J., Yatskevich, S., Rappsilber, J., Hu, B., Nasmyth, K., and Löwe, J. (2019). A folded conformation of MukBEF and cohesin. Nat. Struct. Mol. Biol. 26, 227-236.

Chapard, C., Jones, R., van Oepen, T., Scheinost, J.C., and Nasmyth, K. (2019). Sister DNA Entrapment between Juxtaposed Smc Heads and Kleisin of the Cohesin Complex. Mol. Cell 75, 224-237.e5.

Collier, J.E., Lee, B.G., Roig, M.B., Yatskevich, S., Petela, N.J., Metson, J., Voulgaris, M., Gonzalez Llamazares, A., Löwe, J., and Nasmyth, K.A. (2020). Transport of DNA within cohesin involves clamping on top of engaged heads by Scc2 and entrapment within the ring by Scc3. eLife 9, e59560.

Diebold-Durand, M.L., Lee, H., Ruiz Avila, L.B., Noh, H., Shin, H.C., Im, H., Bock, F.P., Bürmann, F., Durand, A., Basfeld, A., et al. (2017). Structure of Full-Length SMC and Rearrangements Required for Chromosome Organization. Mol. Cell 67, 334-347.e5.

Earnshaw, W.C., and Laemmli, U.K. (1983). Architecture of metaphase chromosomes and chromosome scaffolds. J. Cell Biol. 96, 84-93.

Eeftens, J.M., Katan, A.J., Kschonsak, M., Hassler, M., de Wilde, L., Dief, E.M., Haering, C.H., and Dekker, C. (2016). Condensin Smc2-Smc4 Dimers Are Flexible and Dynamic. Cell Rep. 14, 1813-1818.

El Mkami, H., and Norman, D.G. (2015). EPR Distance Measurements in Deuterated Proteins. Methods Enzymol. 564, 125-152.

Fuentes-Perez, M.E., Gwynn, E.J., Dillingham, M.S., and Moreno-Herrero, F. (2012). Using DNA as a fiducial marker to study SMC complex interactions with the atomic force microscope. Biophys. J. 102, 839-848.

Gibcus, J.H., Samejima, K., Goloborodko, A., Samejima, I., Naumova, N., Nuebler, J., Kanemaki, M.T., Xie, L., Paulson, J.R., Earnshaw, W.C., et al. (2018). A pathway for mitotic chromosome formation. Science 359, eaao6135.

Gligoris, T.G., Scheinost, J.C., Bürmann, F., Petela, N., Chan, K.L., Uluocak, P., Beckouët, F., Gruber, S., Nasmyth, K., and Löwe, J. (2014). Closing the cohesin ring: structure and function of its Smc3-kleisin interface. Science 346, 963-967.

Griese, J.J., and Hopfner, K.P. (2011). Structure and DNA-binding activity of the Pyrococcus furiosus SMC protein hinge domain. Proteins 79, 558-568.

Gruber, S., and Errington, J. (2009). Recruitment of condensin to replication origin regions by ParB/SpoOJ promotes chromosome segregation in B. subtilis. Cell 137, 685-696.
Gruber, S., Veening, J.W., Bach, J., Blettinger, M., Bramkamp, M., and Errington, J. (2014). Interlinked sister chromosomes arise in the absence of condensin during fast replication in B. subtilis. Curr. Biol. 24, 293-298.

Haering, C.H., Löwe, J., Hochwagen, A., and Nasmyth, K. (2002). Molecular architecture of SMC proteins and the yeast cohesin complex. Mol. Cell 9, 773-788.

Hassler, M., Shaltiel, I.A., and Haering, C.H. (2018). Towards a Unified Model of SMC Complex Function. Curr. Biol. 28, R1266-R1281.

Higashi, T.L., Eickhoff, P., Sousa, J.S., Locke, J., Nans, A., Flynn, H.R., Snijders, A.P., Papageorgiou, G., O'Reilly, N., Chen, Z.A., et al. (2020). A Structure-Based Mechanism for DNA Entry into the Cohesin Ring. Mol. Cell 79, $917-$ 933.e9.

Hirano, M., and Hirano, T. (2006). Opening closed arms: long-distance activation of SMC ATPase by hinge-DNA interactions. Mol. Cell 21, 175-186.

Hirano, M., Anderson, D.E., Erickson, H.P., and Hirano, T. (2001). Bimodal activation of SMC ATPase by intra- and inter-molecular interactions. EMBO J. 20, 3238-3250.

Hopfner, K.P. (2016). Invited review: Architectures and mechanisms of ATP binding cassette proteins. Biopolymers 105, 492-504.

Jalal, A.S.B., and Le, T.B.K. (2020). Bacterial chromosome segregation by the ParABS system. Open Biol. 10, 200097.

Jeschke, G. (2012). DEER distance measurements on proteins. Annu. Rev. Phys. Chem. 63, 419-446.

Jeschke, G. (2018). The contribution of modern EPR to structural biology. Emerg. Top. Life Sci. 2, 9-18.

Jeschke, G., Chechik, V., Ionita, P., Godt, A., Zimmermann, H., Banham, J., Timmel, C., Hilger, D., and Jung, H. (2006). DeerAnalysis2006-a comprehensive software package for analyzing pulsed ELDOR data. Appl. Magn. Reson. 30, 473-498.

Kamada, K., Su'etsugu, M., Takada, H., Miyata, M., and Hirano, T. (2017). Overall Shapes of the SMC-ScpAB Complex Are Determined by Balance between Constraint and Relaxation of Its Structural Parts. Structure 25, 603616.e604.

Kazmier, K., Sharma, S., Islam, S.M., Roux, B., and Mchaourab, H.S. (2014) Conformational cycle and ion-coupling mechanism of the $\mathrm{Na}+$ /hydantoin transporter Mhp1. Proc. Natl. Acad. Sci. USA 111, 14752-14757.

Lammens, A., Schele, A., and Hopfner, K.P. (2004). Structural biochemistry of ATP-driven dimerization and DNA-stimulated activation of SMC ATPases. Curr. Biol. 14, 1778-1782.

Lee, B.G., Merkel, F., Allegretti, M., Hassler, M., Cawood, C., Lecomte, L., O'Reilly, F.J., Sinn, L.R., Gutierrez-Escribano, P., Kschonsak, M., et al. (2020). Cryo-EM structures of holo condensin reveal a subunit flip-flop mechanism. Nat. Struct. Mol. Biol. 27, 743-751.

Marko, J.F., De Los Rios, P., Barducci, A., and Gruber, S. (2019). DNAsegment-capture model for loop extrusion by structural maintenance of chromosome (SMC) protein complexes. Nucleic Acids Res. 47, 6956-6972.

Marsden, M.P., and Laemmli, U.K. (1979). Metaphase chromosome structure: evidence for a radial loop model. Cell 17, 849-858.

Merkenschlager, M., and Nora, E.P. (2016). CTCF and Cohesin in Genome Folding and Transcriptional Gene Regulation. Annu. Rev. Genomics Hum. Genet. 17, 17-43.

Minnen, A., Bürmann, F., Wilhelm, L., Anchimiuk, A., Diebold-Durand, M.L., and Gruber, S. (2016). Control of Smc Coiled Coil Architecture by the ATPase Heads Facilitates Targeting to Chromosomal ParB/parS and Release onto Flanking DNA. Cell Rep. 14, 2003-2016.

Muir, K.W., Li, Y., Weis, F., and Panne, D. (2020). The structure of the cohesin ATPase elucidates the mechanism of SMC-kleisin ring opening. Nat. Struct. Mol. Biol. 27, 233-239.

Naumova, N., Imakaev, M., Fudenberg, G., Zhan, Y., Lajoie, B.R., Mirny, L.A., and Dekker, J. (2013). Organization of the mitotic chromosome. Science 342, 948-953. 
Orlandini, E., Marenduzzo, D., and Michieletto, D. (2019). Synergy of topoisomerase and structural-maintenance-of-chromosomes proteins creates a universal pathway to simplify genome topology. Proc. Natl. Acad. Sci. USA $116,8149-8154$.

Osorio-Valeriano, M., Altegoer, F., Steinchen, W., Urban, S., Liu, Y., Bange, G., and Thanbichler, M. (2019). ParB-type DNA Segregation Proteins Are CTPDependent Molecular Switches. Cell 179, 1512-1524.e1515.

Pannier, M., Veit, S., Godt, A., Jeschke, G., and Spiess, H.W. (2000). Deadtime free measurement of dipole-dipole interactions between electron spins. J. Magn. Reson. 142, 331-340.

Polyhach, Y., Bordignon, E., Tschaggelar, R., Gandra, S., Godt, A., and Jeschke, G. (2012). High sensitivity and versatility of the DEER experiment on nitroxide radical pairs at Q-band frequencies. Phys. Chem. Chem. Phys. 14, 10762-10773.

Reginsson, G.W., and Schiemann, O. (2011). Pulsed electron-electron double resonance: beyond nanometre distance measurements on biomacromolecules. Biochem. J. 434, 353-363.

Ryu, J.-K., Katan, A.J., van der Sluis, E.O., Wisse, T., de Groot, R., Haering, C., and Dekker, C. (2019). AFM images of open and collapsed states of yeast condensin suggest a scrunching model for DNA loop extrusion. bioRxiv. https:// doi.org/10.1101/2019.12.13.867358.

Shi, Z., Gao, H., Bai, X.C., and Yu, H. (2020). Cryo-EM structure of the human cohesin-NIPBL-DNA complex. Science 368, 1454-1459.

Soh, Y.M., Bürmann, F., Shin, H.C., Oda, T., Jin, K.S., Toseland, C.P., Kim, C., Lee, H., Kim, S.J., Kong, M.S., et al. (2015). Molecular basis for SMC rod formation and its dissolution upon DNA binding. Mol. Cell 57, 290-303.

Soh, Y.M., Davidson, I.F., Zamuner, S., Basquin, J., Bock, F.P., Taschner, M., Veening, J.W., De Los Rios, P., Peters, J.M., and Gruber, S. (2019). Self-organization of parS centromeres by the ParB CTP hydrolase. Science 366, 11291133.

Sullivan, N.L., Marquis, K.A., and Rudner, D.Z. (2009). Recruitment of SMC by ParB-parS organizes the origin region and promotes efficient chromosome segregation. Cell 137, 697-707.

Terakawa, T., Bisht, S., Eeftens, J.M., Dekker, C., Haering, C.H., and Greene, E.C. (2017). The condensin complex is a mechanochemical motor that translocates along DNA. Science 358, 672-676.
Tran, N.T., Laub, M.T., and Le, T.B.K. (2017). SMC Progressively Aligns Chromosomal Arms in Caulobacter crescentus but Is Antagonized by Convergent Transcription. Cell Rep. 20, 2057-2071.

Vazquez Nunez, R., Ruiz Avila, L.B., and Gruber, S. (2019). Transient DNA Occupancy of the SMC Interarm Space in Prokaryotic Condensin. Mol. Cell 75, 209-223.e6.

Vercellino, I., Rezabkova, L., Olieric, V., Polyhach, Y., Weinert, T., Kammerer R.A., Jeschke, G., and Korkhov, V.M. (2017). Role of the nucleotidyl cyclase helical domain in catalytically active dimer formation. Proc. Natl. Acad. Sci. USA 114, E9821-E9828.

Wang, X., Tang, O.W., Riley, E.P., and Rudner, D.Z. (2014). The SMC condensin complex is required for origin segregation in Bacillus subtilis. Curr. Biol. 24 287-292.

Wang, X., Le, T.B., Lajoie, B.R., Dekker, J., Laub, M.T., and Rudner, D.Z. (2015). Condensin promotes the juxtaposition of DNA flanking its loading site in Bacillus subtilis. Genes Dev. 29, 1661-1675.

Wang, X., Brandão, H.B., Le, T.B., Laub, M.T., and Rudner, D.Z. (2017). BacilIus subtilis SMC complexes juxtapose chromosome arms as they travel from origin to terminus. Science 355, 524-527.

Wang, X., Hughes, A.C., Brandão, H.B., Walker, B., Lierz, C., Cochran, J.C., Oakley, M.G., Kruse, A.C., and Rudner, D.Z. (2018). In Vivo Evidence for ATPase-Dependent DNA Translocation by the Bacillus subtilis SMC Condensin Complex. Mol. Cell 71, 841-847.e5.

Wilhelm, L., Bürmann, F., Minnen, A., Shin, H.C., Toseland, C.P., Oh, B.H., and Gruber, S. (2015). SMC condensin entraps chromosomal DNA by an ATP hydrolysis dependent loading mechanism in Bacillus subtilis. eLife 4, e06659.

Yatskevich, S., Rhodes, J., and Nasmyth, K. (2019). Organization of Chromosomal DNA by SMC Complexes. Annu. Rev. Genet. 53, 445-482.

Yoshimura, S.H., Hizume, K., Murakami, A., Sutani, T., Takeyasu, K., and Yanagida, M. (2002). Condensin architecture and interaction with DNA: regulatory non-SMC subunits bind to the head of SMC heterodimer. Curr. Biol. 12 508-513.

Zhao, S., and Fernald, R.D. (2005). Comprehensive algorithm for quantitative real-time polymerase chain reaction. J. Comput. Biol. 12, 1047-1064. 


\section{STAR $\star M E T H O D S$}

\section{KEY RESOURCES TABLE}

\begin{tabular}{|c|c|c|}
\hline REAGENT or RESOURCE & SOURCE & IDENTIFIER \\
\hline \multicolumn{3}{|l|}{ Antibodies } \\
\hline Anti-Smc polyclonal rabbit antibody, affinity purified & Gruber Lab & COD008 \\
\hline \multicolumn{3}{|l|}{ Chemicals, peptides, and recombinant proteins } \\
\hline Adenosine Triphosphate (ATP) & Jena Bioscience & Cat\#987-65-5 \\
\hline Bis(malemido)ethane (BMOE) & Thermo Scientific & Cat\#22323 \\
\hline Breathe-Easy & Diversified Biotech & Cat\#BEM-1 \\
\hline Bsal & New England Biolabs & Cat\#R0559L \\
\hline Deuterium Oxide & Sigma-Aldrich & Cat\#435767-100G \\
\hline Dynabeads Protein-G & Life Technologies & Cat\#10004D \\
\hline Erythromycin & AppliChem & Cat\#A2275,0005 \\
\hline Ethylene glycol-d6 & Sigma-Aldrich & Cat\#530549 \\
\hline GlycolBlue & Ambion & Cat\#AM9515 \\
\hline HaloTag-TMR Ligand & Promega & Cat\#G8251 \\
\hline HiTrap Blue HP & Cytiva & Cat\#17041301 \\
\hline HiTrap Butyl HP & Cytiva & Cat\#28411005 \\
\hline HiTrap Q HP & Cytiva & Cat\#17115401 \\
\hline HiTrap Heparin HP & Cytiva & Cat\#17040701 \\
\hline HiLoad 16/60 S75 & Cytiva & Cat\#28-9893-33 \\
\hline HiLoad 16/60 S200 pg & Cytiva & Cat\#28989335 \\
\hline Lysozyme From Chicken Egg White & Sigma-Aldrich & Cat\#L6876-5G \\
\hline Lincomycin Hydrochloride Hydrate & Sigma-Aldrich & Cat\#31727-250MG \\
\hline MTSL & Santa Cruz & Cat\#sc-208677 \\
\hline Nicotinamide Adenine Dinucleotide (NADH) & Santa Cruz & Cat\#205762A \\
\hline NuPAGE LDS Sample Buffer (4X) & Life Technologies & Cat\#NP0008 \\
\hline Phosphoenolpyruvic Acid (PEP) & Sigma-Aldrich & Cat\#P7002-100MG \\
\hline Phusion HotStart II DNA Polymerase & Thermo Scientific & Cat\#F-549L \\
\hline Protease Inhibitor Cocktail & Sigma-Aldrich & Cat\#P8849-5ML \\
\hline Pyruvate Kinase/Lactate Dehydrogenase & Sigma-Aldrich & Cat\#P0294-5ML \\
\hline Ready-Lyse Lysozyme Solution & Epicenter & Cat\#R1802M \\
\hline RNase A & Sigma-Aldrich & Cat\#R5125 \\
\hline Sm DNase & MPIB Core Facility & SmDNase \\
\hline Superose 6 increase 10/300 & Cytiva & Cat\#29091596 \\
\hline Superose 6 pg XK 16/70 & Cytiva & Cat\#90100042 \\
\hline TEMPOL & Sigma-Aldrich & Cat\#581500-500MG \\
\hline Tris(2-carboxyethyl) Phosphine Hydrochloride Solution (TCEP) & Sigma-Aldrich & Cat\#646547 \\
\hline T4 DNA Ligase & Thermo Scientific & Cat\#EL0016 \\
\hline \multicolumn{3}{|l|}{ Critical commercial assays } \\
\hline MasterBlock 96 well, $2 \mathrm{~mL}$ & Greiner Bio One & Cat\#780270 \\
\hline Takyon SYBR 2xMasterMix Blue dTTP No ROX & Eurogentec & Cat\#UF-NSMT-B0701 \\
\hline Novex $4-12 \%$ Tris-Glycine Mini Gels & Life Technologies & Cat\#XP04125BOX \\
\hline NuPAGE 3-8\% Tris-Acetate Gels & Life Technologies & Cat\#EA03755BOX \\
\hline QIAquick PCR Purification Kit & QIAGEN & Cat\#28106 \\
\hline Zeba Spin Desalting Column, 7K MWCO, 0.5 mL & Thermo Scientific & Cat\#89882 \\
\hline
\end{tabular}




\begin{tabular}{|c|c|c|}
\hline Continued & & \\
\hline REAGENT or RESOURCE & SOURCE & IDENTIFIER \\
\hline \multicolumn{3}{|l|}{ Deposited data } \\
\hline Original data & Mendeley Data & https://doi.org/10.17632/2g37f3mvbb.1 \\
\hline \multicolumn{3}{|l|}{ Experimental models: Organisms/strains } \\
\hline E. coli and B. subtilis strains, see Table S5 & Gruber Lab & N/A \\
\hline \multicolumn{3}{|l|}{ Oligonucleotides } \\
\hline parS-359 Fw for qPCR & Gruber Lab & N/A \\
\hline \multicolumn{3}{|l|}{ AAAAAGTGATTGCGGAGCAG } \\
\hline parS-359 Rv for qPCR & Gruber Lab & $\mathrm{N} / \mathrm{A}$ \\
\hline \multicolumn{3}{|l|}{ AGAACCGCATCTTTCACAGG } \\
\hline dnaA Fw for qPCR & Gruber Lab & $N / A$ \\
\hline \multicolumn{3}{|l|}{ GATCAATCGGGGAAAGTGTG } \\
\hline dnaA Rv for qPCR & Gruber Lab & $\mathrm{N} / \mathrm{A}$ \\
\hline \multicolumn{3}{|l|}{ GTAGGGCCTGTGGATTTGTG } \\
\hline yocGH Fw for qPCR & Gruber Lab & $N / A$ \\
\hline \multicolumn{3}{|l|}{ TCCATATCCTCGCTCCTACG } \\
\hline yocGH Rv for qPCR & Gruber Lab & N/A \\
\hline \multicolumn{3}{|l|}{ АTTCTGCTGATGTGCAATGG } \\
\hline $40 \mathrm{bp}$ random sequence DNA $\mathrm{A}$ & Gruber Lab & N/A \\
\hline \multicolumn{3}{|l|}{ TTAGTTGTTCGTAGTGCTCGTCTGGCTCTGGATTACCCGC } \\
\hline 40 bp random sequence DNA B & Gruber Lab & N/A \\
\hline \multicolumn{3}{|l|}{ GCGGGTAATCCAGAGCCAGACGAGCACTACGAACAACTAA } \\
\hline \multicolumn{3}{|l|}{ Recombinant DNA } \\
\hline Plasmid DNA, see Table S6 & Gruber Lab & $\mathrm{N} / \mathrm{A}$ \\
\hline \multicolumn{3}{|l|}{ Software and algorithms } \\
\hline Real Time PCR Miner & Zhao and Fernald, 2005 & http://ewindup.info/miner/ \\
\hline GraphPad Prism 8 & GraphPad Software & https://www.graphpad.com:443/ \\
\hline ImageQuant TL 1D V8.1 & GE Healthcare & $\begin{array}{l}\text { https://www.cytivalifesciences.com/en/ } \\
\text { us/shop/protein-analysis/molecular- } \\
\text { imaging-for-proteins/imaging-software/ } \\
\text { imagequant-tl-8-1-p-00110 }\end{array}$ \\
\hline MATLAB 2018a & MathWorks & https://www.mathworks.com \\
\hline DeerAnalysis package & EPR-ETHZ & https://www.epr.ethz.ch/software/index \\
\hline
\end{tabular}

\section{RESOURCE AVAILABILITY}

Lead contact

Further information and requests for resources and reagents should be directed to and will be fulfilled by the Lead Contact, Stephan Gruber (stephan.gruber@unil.ch).

Materials availability

All unique reagents generated in this study are available from the Lead Contact without restrictions.

Data and code availability

Original raw and source data used for generating figures in this paper are available at Mendeley Data (https://doi.org/10.17632/ 2g37f3mvbb.1).

\section{EXPERIMENTAL MODEL AND SUBJECT DETAILS}

Bacillus subtilis strains and growth

Bsu strains used in this work are derived from the 1A700 isolate. Genotypes and strain numbers are listed in Table S5. Strain usage is detailed in Table S1. Naturally competent Bsu cells were transformed as in Diebold-Durand et al. (2017) including a longer starvation 
incubation time ( 2 hours) for high efficiency. The transformants were selected on SMG-agar plates with appropriate antibiotics and single-colonies were isolated. The strains were confirmed by PCR and Sanger sequencing as required. For dilution spot assays, cells were grown for 8 hours at $37^{\circ} \mathrm{C}$ in SMG medium and then were diluted to 81 and 59000 -fold. Afterward, dilutions were spotted onto ONA (16 $\mathrm{h}$ incubation) or SMG (24 h incubation) agar plates at $37^{\circ} \mathrm{C}$ (Vazquez Nunez et al., 2019).

\section{Protein purification}

\section{Full-length Bsu Smc}

Native Smc proteins were purified following the procedure described in Bürmann et al. (2017). E. coli BL21-Gold (DE3) strain was transformed with pET-22 or pET-28 derived plasmids containing the $s m c$ recombinant sequences. Proteins were expressed using ZYM-5052 autoinduction medium for $23 \mathrm{~h}$ at $24^{\circ} \mathrm{C}$. Cells were resuspended in lysis buffer $(50 \mathrm{mM} \mathrm{Tris-} \mathrm{HCl} \mathrm{pH} 7.5,150 \mathrm{mM} \mathrm{NaCl}$, $1 \mathrm{mM}$ EDTA, $1 \mathrm{mM} \mathrm{DTT}, 10 \%(\mathrm{w} / \mathrm{v})$ sucrose) supplemented with protease inhibitor cocktail (PIC) and sonicated. The lysate was centrifuged, the supernatant was filtered with a $0.45 \mu \mathrm{M}$ pore size membrane and then loaded onto two HiTrap Blue HP $5 \mathrm{~mL}$ columns connected in series and eluted with lysis buffer containing $1 \mathrm{M} \mathrm{NaCl}$. The main peak elution fractions where diluted in salt-less buffer (50 mM Tris-HCl pH 7.5, $1 \mathrm{mM}$ EDTA, $1 \mathrm{mM} \mathrm{DTT}$ ) to a conductivity equivalent of $50 \mathrm{mM} \mathrm{NaCl}(\approx 8 \mathrm{mS} / \mathrm{cm})$. The diluted sample was supplemented with PIC and loaded on a HiTrap Heparin HP $5 \mathrm{~mL}$ column. Elution was performed by applying a linear gradient of buffer up to $2 \mathrm{M} \mathrm{NaCl}$. The main peak fractions (aprox. $5 \mathrm{~mL}$ ) were collected and further purified by gel filtration on a XK 16/70 Superose 6 PG column in $50 \mathrm{mM}$ Tris- $\mathrm{HCl}$ pH 7.5, $200 \mathrm{mM} \mathrm{NaCl}, 1 \mathrm{mM}$ EDTA, $1 \mathrm{mM}$ TCEP. Main peak fractions where collected, concentrated with a Vivaspin $1510 \mathrm{~K}$ MWCO filter, flash frozen with liquid nitrogen and stored at $-80^{\circ} \mathrm{C}$. Protein concentration was calculated by absorbance using theoretical molar absorption and molecular weight values.

ScpA

Native ScpA was purified following the procedure reported in Vazquez Nunez et al. (2019). The protein was expressed using E. coli BL21-Gold (DE3) transformed with a pET-28 derived plasmid containing ScpA coding sequence. Cells were cultivated in ZYM-5052 autoinduction medium at $16^{\circ} \mathrm{C}$ for $28 \mathrm{~h}$, harvested and resuspended in lysis buffer $(50 \mathrm{mM} \mathrm{Tris}-\mathrm{HCl} \mathrm{pH} \mathrm{7.5,200} \mathrm{mM} \mathrm{NaCl,} 5 \%$ glycerol) supplemented with PIC. After sonication and centrifugation, the supernatant was applied to a $5 \mathrm{~mL} H \mathrm{HiTrapQ}$ ion exchange column and eluted with a gradient up to $2 \mathrm{M} \mathrm{NaCl}$. The peak fractions were mixed with $4 \mathrm{M} \mathrm{NaCl}$ buffer to reach a final concentration of $3 \mathrm{M} \mathrm{NaCl}$. The sample was injected into a HiTrap Butyl HP column and eluted in a reverse gradient to $50 \mathrm{mM} \mathrm{NaCl}$. Peak fractions were pooled and concentrated to $5 \mathrm{~mL}$ in Vivaspin $1510 \mathrm{~K}$ MWCO filters and subsequently purified by gel filtration in a Hi Load 16/600 Superdex $75 \mathrm{pg}$ column, equilibrated in $20 \mathrm{mM}$ Tris- $\mathrm{HCl}$ pH 7.5, $200 \mathrm{mM} \mathrm{NaCl}$. Protein was concentrated, aliquoted, flash frozen and stored at $-80^{\circ} \mathrm{C}$.

$\operatorname{ScpB}$

Native ScpB was purified using the procedure described in Vazquez Nunez et al. (2019). A pET-22 derived plasmid with the coding sequence of ScpB was transformed in chemically competent $E$. coli cells. Cells were cultivated I ZYM-5052 autoinduction medium at $24^{\circ} \mathrm{C}$ for $23 \mathrm{~h}$. Cells were harvested and resuspended in lysis buffer (50 mM Tris-HCl pH 7.5, $150 \mathrm{mM} \mathrm{NaCl}, 1 \mathrm{mM}$ EDTA, $\left.1 \mathrm{mM} \mathrm{DTT}\right)$ supplemented with PIC. After sonication and centrifugation, the supernatant was diluted to $50 \mathrm{mM} \mathrm{NaCl}$, loaded onto a $5 \mathrm{~mL} \mathrm{HiTrap} \mathrm{Q}$ $\mathrm{HP}$ column and eluted with a gradient to $2 \mathrm{M} \mathrm{NaCl}$. The sample was diluted in lysis buffer with $4 \mathrm{M} \mathrm{NaCl}$ buffer in order to reach a final concentration of $3 \mathrm{M} \mathrm{NaCl}$. The sample was applied on two $5 \mathrm{~mL} \mathrm{HiTrap} \mathrm{Butyl} \mathrm{column} \mathrm{connected} \mathrm{in} \mathrm{series.} \mathrm{Protein} \mathrm{was} \mathrm{eluted} \mathrm{with} \mathrm{a}$ reverse gradient to $50 \mathrm{mM} \mathrm{NaCl}$. A second run on the $5 \mathrm{~mL} \mathrm{HiTrap} \mathrm{Q} \mathrm{HP} \mathrm{column} \mathrm{in} \mathrm{the} \mathrm{same} \mathrm{conditions} \mathrm{as} \mathrm{described} \mathrm{above} \mathrm{was}$ necessary in some cases when the sample still showed $260 / 280$ ratios close to 1 . The peak fractions were concentrated and further purified by SEC using a Hi Load 16/600 Superdex 200 pg equilibrated in $50 \mathrm{mM}$ Tris-HCl pH 7.5, $100 \mathrm{mM} \mathrm{NaCl,} 1 \mathrm{mM} \mathrm{DTT}$. The fractions containing the protein were concentrated, aliquoted, flash frozen and stored at $-80^{\circ} \mathrm{C}$.

\section{METHOD DETAILS}

\section{ATPase assay}

ATPase activity measurements were done using the coupled reaction of pyruvate kinase/lactate dehydrogenase (Bürmann et al., 2017). ADP production was monitored for $1 \mathrm{~h}$ by oxidation of NADH absorbance changes at $340 \mathrm{~nm}$. The values were collected using a Synergy Neo Hybrid Multi-Mode Microplate reader. The reaction mixture consisted in $1 \mathrm{mM}$ NADH, 3 mM Phosphoenol pyruvic acid, $100 \mathrm{U}$ Pyruvate kinase, $20 \mathrm{U}$ Lactate dehydrogenase and the appropriate ATP concentrations. Double-stranded oligonucleotides (40 bp (5'-TTAGTTGTTC GTAGTGCTCG TCTGGCTCTG GATTACCCGC)) were added for measurements that required it to a final concentration of $3 \mu \mathrm{M}$, unless indicated differently. The final protein concentration in the assay was $0.15 \mu \mathrm{M}$ Smc dimers in ATPase assay buffer (50 mM HEPES- $\mathrm{KOH} \mathrm{pH} \mathrm{7.5,50} \mathrm{mM} \mathrm{NaCl,} 2 \mathrm{mM} \mathrm{MgCl}$ ). Measurements were carried out at $25^{\circ} \mathrm{C}$.

\section{M3M crosslinking-ATPase assay}

For this assay, a fresh full-length Smc purification (as described above) was done for each experiment. The last SEC purification step, however, was done in $50 \mathrm{mM}$ Tris- $\mathrm{HCl} \mathrm{pH} \mathrm{7.5,200} \mathrm{mM} \mathrm{NaCl}$ in order to remove any trace of reducing agents. The peak fractions were concentrated to $\sim 35 \mu \mathrm{M}$ Smc dimer in a Vivaspin $1510 \mathrm{~K}$ MWCO filters. The crosslinked reaction was carried on by mixing 10-fold thiol molar excess of M3M (diluted in DMSO). A typical reaction was done in $500 \mu \mathrm{L}$ with $30 \mu \mathrm{M}$ Smc dimers and $600 \mu \mathrm{M}$ M3M in $50 \mathrm{mM}$ Tris- $\mathrm{HCl} \mathrm{pH} \mathrm{7.5,} 200 \mathrm{mM} \mathrm{NaCl}$ at $4^{\circ} \mathrm{C}$ overnight, protected from light. The final concentration sometimes differed slightly, 
depending on the purification yield of the single cysteine mutant, but the M3M:thiol ratio was always maintained. A negative control was always included by mixing the same amount of protein with an equivalent volume of DMSO as in the experimental M3M reaction.

After crosslinking the sample was centrifuged to remove big aggregates and the excess of crosslinker was removed with a Zeba spin 7K MWCO $0.5 \mathrm{~mL}$ desalting column, preequilibrated with $50 \mathrm{mM}$ Tris- $\mathrm{HCl}$ pH 7.5, $200 \mathrm{mM} \mathrm{NaCl}$. An additional gel filtration purification was done to remove small aggregates and traces of crosslinker in a Superose 6 increase 10/300 column equilibrated in $50 \mathrm{mM}$ Tris- $\mathrm{HCl} \mathrm{pH} \mathrm{7.5,} 200 \mathrm{mM} \mathrm{NaCl}$. Afterward, the fractions at the maximum of the peak were recovered. The concentration of the protein was typically $\sim \mu \mathrm{M}$ Smc dimer. The sample was mixed with ScpA and ScpB and the ATPase assay was performed as described above. $10 \mathrm{mM}$ DTT final concentration was added for the samples that required it.

Fluorescence anisotropy measurements

Fluorescence anisotropy was measured using a 40 bp dsDNA (as described in ATPase assay) modified at the $3^{\prime}$ end with fluorescein. Measurements were recorded using a Synergy Neo Hybrid Multi-Mode Microplate reader (BioTek) with the appropriate filters in black 96-well flat bottom plates at $25^{\circ} \mathrm{C}$. Buffer conditions were $50 \mathrm{mM}$ Tris- $\mathrm{HCl} \mathrm{pH} \mathrm{7.5,50} \mathrm{mM} \mathrm{NaCl,} 2 \mathrm{mM} \mathrm{MgCl} 250 \mathrm{nM}$ dsDNA and $1 \mathrm{mM}$ ATP for all measurements. Anisotropy measurements where exported from the BioTek Synergy Neo software and subsequently fit to a binding polynomial using non-linear regression in GraphPad Prism 8 (Vazquez Nunez et al., 2019)

Double electron-electron resonance (EPR-DEER)

$\mathrm{Smc}^{3 \mathrm{C}}$, ScpA and ScpB purifications were performed as described above, except for the last gel filtration step of Smc purification which was performed in absence of reducing agent. In order to avoid premature thiol oxidation, the sample was mixed with 10-fold molar excess of MTSL per thiol, immediately after the last purification step. The reaction volume was $500 \mu \mathrm{L}$ and consisted in $\sim 40 \mu \mathrm{M}$ Smc dimer and $800 \mu \mathrm{M} \mathrm{MTSL}$ in $50 \mathrm{mM}$ Tris- $\mathrm{HCl} \mathrm{pH} 7.5$ at $4^{\circ} \mathrm{C}$ and $200 \mathrm{mM} \mathrm{NaCl}$. The reaction was incubated over-night at $4{ }^{\circ} \mathrm{C}$ protected from light. After the incubation, the sample was centrifuged to remove big aggregates and the MTSL excess was removed using a Zeba Spin desalting column, $7 \mathrm{~K} \mathrm{MWCO}, 0.5 \mathrm{~mL}$. Afterward the sample was further purified by gel filtration in a Superose $610 /$ 300 increase, equilibrated with $50 \mathrm{mM}$ Tris- $\mathrm{HCl} \mathrm{pH} 7.5$ at $4{ }^{\circ} \mathrm{C}, 200 \mathrm{mM} \mathrm{NaCl}$ and $90 \%$ deuterium oxide (heavy water). The sample was concentrated to $\sim 20 \mu \mathrm{M}$ Smc dimer and the label efficiency was determined by calculating the double integral of the continuous wave (CW) EPR spectrum. Room temperature CW EPR measurements were done at X band ( 9.7 GHz) using a commercial X-band Magnettech MiniScope MS 400. Spectra were taken at $13 \mathrm{~dB}$ attenuation, corresponding to $5 \mathrm{~mW}$ incident microwave power and $0.15 \mathrm{mT}$ magnetic field modulation amplitude. Samples were placed into $1 \mathrm{~mm}$ o.d. glass capillaries. Spin labeling efficiency for all mutants was estimated by comparing double integrals of EPR signals from the Smc mutants and the concentration standard (200 $\mu \mathrm{M}$ water solution of TEMPOL).

Samples were flash frozen and stored at $-80^{\circ} \mathrm{C}$ until use. Before measurement, the protein samples $(\mathrm{Smc}, \mathrm{ScpA}$ and $\mathrm{ScpB})$ were thawed and centrifuged to remove big aggregates. The final concentration per condition were: $5 \mu \mathrm{M} \mathrm{SmcScpAB}, 10 \mu \mathrm{M}$ dsDNA 40 bp and $1 \mathrm{mM}$ ATP in $50 \mathrm{mM}$ Tris- $\mathrm{HCl} \mathrm{pH} 7.5$ at $4{ }^{\circ} \mathrm{C}, 50 \mathrm{mM} \mathrm{NaCl}, 2 \mathrm{mM} \mathrm{MgCl}$, $40 \%$ Ethylene glycol-d6 (as cryoprotectant) and $90 \%$ deuterium oxide. Samples were incubated 5 minutes in $1.5 \mathrm{~mL}$ centrifuge tubes at $25^{\circ} \mathrm{C}$ with gentile $(700$ rpm) shaking. Distance measurements were performed at Q-band frequencies ( $34.4 \mathrm{GHz})$ using a standard double electron-electron resonance (DEER) sequence (Pannier et al., 2000). DEER traces were acquired at $50 \mathrm{~K}$. A commercial X/Q-band Elexsys E580 spectrometer (Bruker) power-upgraded to $200 \mathrm{~W}$ equipped with the homebuilt TE102 rectangular resonator was used (Polyhach et al., 2012). The protein solution ready for measurement was filled into the $3 \mathrm{~mm}$ o.d. quartz tubes, shock-frozen by immersion into liquid nitrogen and inserted into the pre-cooled resonator. All observer pulses were $16 \mathrm{~ns}$ long, the pump pulse was $12 \mathrm{~ns}$ long, the pump frequency was set at the global maximum of the nitroxide EPR spectrum and the observer frequency was set $100 \mathrm{MHz}$ lower.

In vivo cross-linking

Bsu cultures in $200 \mathrm{~mL}$ SMG were grown to exponential phase $\left(\mathrm{OD}_{600}=0.02\right)$ at $37^{\circ} \mathrm{C}$. Cells were collected by filtration harvesting and washed in cold PBS $+0.1 \%(\mathrm{v} / \mathrm{v})$ glycerol ('PBSG'). A biomass equivalent to $0.85 \mathrm{OD}$ units was sorted. Cells samples were centrifuged 2 min at 10,000 g, resuspended in fresh PBSG $(200 \mu \mathrm{L})$. The cross-linking reaction was started by adding $0.5 \mathrm{mM}$ BMOE and it was incubated for $10 \mathrm{~min}$ on ice. The reaction was quenched by the addition of $1.4 \mathrm{mM} 2$-mercaptoethanol. Cells were centrifuged and resuspended in $30 \mu \mathrm{L}$ of lysis mix $(75 \mathrm{U} / \mathrm{mL}$ ReadyLyse Lysozyme, $750 \mathrm{U} / \mathrm{mL}$ Sm DNase, $5 \mu$ M HaloTag TMR Substrate and protease inhibitor cocktail 'PIC' in PBSG). Lysis reaction was incubated at $37^{\circ} \mathrm{C}$ for 30 min. Afterward, the material was mixed with $10 \mu \mathrm{L}$ of $4 \mathrm{X}$ LDS-PAGE buffer, samples were incubated for $5 \mathrm{~min}$ at $95^{\circ} \mathrm{C}$ and resolved by SDS-PAGE. Gels were imaged on an Amersham Typhoon scanner with Cy3 DIGE filter setup.

\section{Chromatin immunoprecipitation}

The procedure followed the one described in Vazquez Nunez et al. (2019). Bsu strains were cultured in $200 \mathrm{~mL}$ SMG medium from OD600 $=0.004$ initial OD to OD600 $=0.02$ at $37^{\circ} \mathrm{C}$. Cells were crosslinked with $20 \mathrm{~mL}$ of buffer $\mathrm{F}(50 \mathrm{mM} \mathrm{Tris-} \mathrm{HCl} \mathrm{pH} 7.4,100 \mathrm{mM}$ $\mathrm{NaCl}, 0.5 \mathrm{mM}$ EGTA pH 8.0, $1 \mathrm{mM}$ EDTA pH 8.0, 10\% (w/v) formaldehyde) and incubated for 30 min at room temperature with occasional manual shaking. Cells were harvested by filtration and washed in PBS at $4^{\circ} \mathrm{C}$. The cell biomass equivalent to $2 \mathrm{OD}_{600}$ units was resuspended in $1 \mathrm{~mL}$ TSEMS $(50 \mathrm{mM}$ Tris $\mathrm{pH} 7.4,50 \mathrm{mM} \mathrm{NaCl}, 10 \mathrm{mM}$ EDTA pH 8.0, $0.5 \mathrm{M}$ sucrose and protease inhibitor cocktail) containing $6 \mathrm{mg} / \mathrm{mL}$ lysozyme from chicken egg white. Primary lysis was done at $37^{\circ} \mathrm{C}$ for 30 min with shaking at 
$1400 \mathrm{rpm}$. Protoplasts were washed twice and resuspended in $1 \mathrm{~mL}$ TSEMS. The sample then was split into 3 aliquots and pelleted. Pellets were flash frozen and stored at $-80^{\circ} \mathrm{C}$ until used.

Each pellet was thawed and resuspended in $2 \mathrm{~mL}$ buffer $\mathrm{L}(50 \mathrm{mM} \mathrm{HEPES}-\mathrm{KOH} \mathrm{pH}$ 7.5, $140 \mathrm{mM} \mathrm{NaCl}, 1 \mathrm{mM}$ EDTA pH 8.0, $1 \%$ (v/v) Triton X-100, 0.1\% (w/v) Na-deoxycholate) containing $0.1 \mathrm{mg} / \mathrm{mL}$ RNase A and PIC. The suspension was sonicated during $1 \mathrm{~min}$ in 3 cycles of $20 \mathrm{~s}$ and $40 \%$ amplitude at $4^{\circ} \mathrm{C}$ using a MS73 probe. The sonicated material was centrifuged at $4{ }^{\circ} \mathrm{C}$ and $20,000 \times \mathrm{g}$ and $200 \mu \mathrm{L}$ of the supernatant were collected as input reference. The immunoprecipitation was carried out by mixing $750 \mu \mathrm{L}$ of the extract with $50 \mu \mathrm{L}$ of Dynabeads Protein-G suspension freshly charged with $50 \mu \mathrm{L} \alpha$-ScpB antiserum and incubated for $2 \mathrm{~h}$ on a wheel at $4{ }^{\circ} \mathrm{C}$. Beads were washed with $1 \mathrm{~mL}$ each of buffer L, buffer L5 (buffer L containing $500 \mathrm{mM} \mathrm{NaCl}$ ), buffer W (10 mM Tris-HCl pH 8.0 , $250 \mathrm{mM} \mathrm{LiCl}, 0.5 \%$ (v/v) NP-40, 0.5\% (w/v) Na-Deoxycholate, $1 \mathrm{mM}$ EDTA pH 8.0) and buffer TE (10 mM Tris-HCl pH 8.0, $1 \mathrm{mM}$ EDTA pH 8.0). All wash steps were done at $25^{\circ} \mathrm{C}$ during 5 min shaking (1400 rpm). Beads were resuspended in $520 \mu \mathrm{L}$ buffer TES (50 mM Tris- $\mathrm{HCl} \mathrm{pH} 8.0,10 \mathrm{mM}$ EDTA pH 8.0, 1\% (w/v) SDS). The reference sample was mixed with, $300 \mu \mathrm{L}$ buffer TES and $20 \mu \mathrm{L} 10 \%$ SDS. Formaldehyde cross-links were reversed over-night at $65^{\circ} \mathrm{C}$ with vigorous shaking.

For phenol/chloroform DNA extraction, samples were cooled to room temperature, mixed with $500 \mu \mathrm{L}$ phenol equilibrated with buffer (10 mM Tris-HCl pH 8.0, $1 \mathrm{mM}$ EDTA), emulsified and centrifuged for $10 \mathrm{~min}$ at 20,000 $\times \mathrm{g}$. Subsequently, $450 \mu \mathrm{L}$ of the supernatant was emulsified with $450 \mu \mathrm{L}$ chloroform and centrifuged for $10 \mathrm{~min}$ at 20,000 $\times \mathrm{g}$. DNA precipitation was done by taking $400 \mu \mathrm{L}$ of the supernatant and mixing with $1.2 \mu \mathrm{L}$ GlycoBlue, $40 \mu \mathrm{L}$ of $3 \mathrm{M} \mathrm{Na}$-Acetate $\mathrm{pH} 5.2$ and $1 \mathrm{~mL}$ ethanol and incubated for $20 \mathrm{~min}$ at $-20^{\circ} \mathrm{C}$. Samples were centrifuged at room temperature and $20,000 \times \mathrm{g}$ for $10 \mathrm{~min}$, and the precipitate was dissolved in $100 \mu \mathrm{L}$ buffer PB (QIAGEN) for $10 \mathrm{~min}$ at $55^{\circ} \mathrm{C}$, purified with a PCR purification kit, and eluted in $50 \mu \mathrm{L}$ buffer EB.

For qPCR, samples were diluted in DNase-free water (1:10 for IP and 1:1000 for input) and $10 \mu \mathrm{L}$ reactions (5 $\mu \mathrm{L}$ Takyon SYBR MasterMix, $1 \mu \mathrm{L}$ of $3 \mu \mathrm{M}$ primer mix, $4 \mu \mathrm{L}$ sample) were run in duplicates in a Rotor-Gene $\mathrm{Q}$ machine (QIAGEN) using the appropriate primer pairs.

\section{QUANTIFICATION AND STATISTICAL ANALYSIS}

\section{Analysis of cross-linking efficiencies}

TMR fluorescence or Coomassie stained bands were quantified using ImageQuant TL 1D V8.1. Lanes were defined manually. Bands were detected automatically, and the band intensities corrected for background signal using the Rolling Ball algorithm with a ball radius set to 129. Values from three replicate experiments were exported to Microsoft Excel for calculation of average fractions and standard deviation.

\section{Steady-state kinetics}

The absorbance values were exported and fit to a straight-line equation in GraphPad prism 8. The slope values were transformed to rate values using the molar absorption coefficient of $\mathrm{NADH}$. The rates were expressed into absolute values by correcting for the protein concentration. When an ATP titration was done, the rates were fit using non-linear regression to the Hill equation:

$$
\frac{V_{\max }[A T P]^{h}}{K_{0.5}^{h}+[A T P]^{h}}
$$

Where $V_{\max }$ is the maximal rate at a given protein concentration, [ATP] is the variable ATP concentration, $K_{0.5}$ is the semi-saturation concentration and $h$ is the Hill coefficient.

Fit parameters obtained from the average of duplicates summarized in Table S4.

\section{DEER traces analysis}

Experimental DEER traces were processed with the open-source DeerAnalysis package (Jeschke et al., 2006), available at https://www.epr.ethz.ch/software/index. For background correction, a homogeneous distribution of spins in 3D space was assumed in all cases. Distance distributions for all mutants were calculated using double Gaussian model, whereas preliminary (calibrating) analysis for one of the mutants (D193) was performed model-free with the Tikhonov regularization.

\section{Analysis of qPCR Data}

The threshold cycle (TC) was obtained by analyzing the fluorescence raw data in the Real-Time PCR Miner server (http://ewindup. info/miner/; Zhao and Fernald, 2005).

$\mathrm{IP} /$ input ratios were calculated as $\alpha 2 \Delta \mathrm{CT}$, where $\Delta \mathrm{CT}=\mathrm{CT}$ (Input) $-\mathrm{CT}$ and $\alpha$ is a constant determined by extraction volumes and sample dilutions. Data are presented as the mean of duplicates. 